\title{
Isotope Effects on the Vaporization of Organic Compounds from an Aqueous Solution - Insight from Experiment and Computations
}

Michał Rostkowski, ${ }^{\mathrm{a}}$ Heide K. V. Schürner, ${ }^{\mathrm{b}}$ Agata Sowińska, ${ }^{\mathrm{a}}$ Luis Vasquez, ${ }^{\mathrm{a}}$ Martyna Przydacz, ${ }^{\text {a }}$ Martin Elsner, ${ }^{\mathrm{b}}$ and Agnieszka Dybala-Defratyka ${ }^{\mathrm{a},{ }^{*}}$

${ }^{a}$ Institute of Applied Radiation Chemistry, Faculty of Chemistry, Lodz University of Technology, Zeromskiego 116, 90-924, Lodz, Poland

${ }^{b}$ Chair of Analytical Chemistry and Water Chemistry, Technical University of Munich, Elisabeth-Winterhalter-Weg 6, 81377 Munich, Germany

*agnieszka.dybala-defratyka@p.lodz.pl 
Theoretical Consideration to Exclude the Possibility of Deuterium Exchange in Partitioning Experiments with Chloroform

To assess the potential for deuterium exchange at room temperature and $\mathrm{pH} \mathrm{7,} \mathrm{we} \mathrm{made} \mathrm{use} \mathrm{of}$ literature data on alkaline hydrogen exchange of chloroform (control experiment in ref. 1). Assuming second-order kinetics with respect to chloroform and $\mathrm{OH}^{-}$, a reported rate of 0.03 per hour at an aqueous $\mathrm{OH}^{-}$concentration of $44 \mathrm{M}$ would correspond to a rate of $7 \cdot 10^{-11}$ per hour (or $1.7 \cdot 10^{-9}$ per day) at an aqueous $\mathrm{OH}^{-}$concentration of $10^{-7} \mathrm{M}$. However, this would be too slow by a factor of $10^{7}$ to explain the observed changes in $\mathrm{D} / \mathrm{H}$ of 10 per mille (or $1 \cdot 10^{-2}$ ) observed in the course of the experiment.

\section{Full explicit solvation models}

Calculations. Minimization was performed in five consecutive steps in which the force constant keeping the solute in place was varying from 500 to $50 \mathrm{kcal} \cdot \mathrm{mol}^{-1} \AA^{-2}$ using the steepest descent and the conjugate gradient methods. Next, the systems were heated from 100 to $300 \mathrm{~K}$ during 100 ps using the NVT ensemble with a time step of 1 fs and equilibrated using the NPT ensemble. The production runs were performed using the NPT ensemble with a time step of $1 \mathrm{fs}$ and the length of the simulations was equal to $80 \mathrm{~ns}$. Periodic boundary conditions with the particle mesh Ewald method ${ }^{2}$ and $10 \AA$ cut-off distance for nonbonding interactions were applied throughout the simulations. After classical MM MD simulations, 10 structures from each trajectory from the range of 20-80 ns were selected and subjected to QM/MM optimization at a DFT/MM level with the QM part consisting of all atoms of the solutes. Combination of a functional and basis set for the DFT/MM optimization was chosen based on the results obtained from the QM cluster calculations; for TCM and TEA it was B3LYP/6-31+G(d,p) and for benzene it was B2PLYP/6-31+G(d,p). The rest of the systems was described using the AMBER force field and the TIP3P potential implemented in fDynamo. ${ }^{3}$ The optimization was performed as implemented in the fDynamo library ${ }^{4}$ and its interface with Gaussian 09 (ver. C01 in case of TCM and E01 for benzene and TEA). Positions of all atoms outside $18 \AA$ sphere were kept fixed during the geometry optimization. $10 \AA$ cut-off distance for nonbonding interactions and 0.4 $\mathrm{kcal} \cdot \mathrm{mol}^{-1} \cdot \AA^{-1}$ gradient tolerance were applied for the optimization purposes. Within every step 
of the calculation a single-point gradient evaluation was performed, and the vibrational Hessian was determined for each properly converged structure.

\section{Tables:}

Table S1. Volume of stock solutions and different equilibration times of benzene, triethylamine, and trichloromethane for water-air and hexadecane-air partitioning, respectively.

\begin{tabular}{l|ccc|ccc} 
& \multicolumn{2}{|c|}{ volume of stock solution [mL] } & \multicolumn{3}{c}{ equilibration time [min] } \\
& benzene & TEA & $\mathbf{C H C l}_{3}$ & benzene & TEA & $\mathbf{C H C l}_{3}$ \\
\hline water-air & 2.80 & 0.07 & 1.00 & 30 & 60 & 30
\end{tabular}

\section{Data Evaluation of Stepwise Partitioning}

Data evaluation was carried out using both, the classical Rayleigh approach (eq. 8), and the following equations for stepwise partitioning as described in detail by Jeannottat et $a l .{ }^{5}$ In short, the following general equation applies for each equilibration step $n$

$\delta^{h} E_{\text {liquid }, n+1}=\delta^{h} E_{\text {liquid }, n} \times f_{\text {liquid }}+\delta^{h} E_{H S, n} \times f_{H S}$

where $f_{H S}$ and $f_{\text {liquid, }}$, respectively, are the fractions of compound in the respective phase and $\delta^{h} E_{n+1}$ and $\delta^{h} E_{n}$ are the isotopic signatures before and after equilibration, respectively. Substitution of $\delta^{h} E_{\text {liquid }}$ by

$\varepsilon \approx \delta^{h} E_{H S}-\delta^{h} E_{\text {liquid }}$

and rearrangement gives the isotopic shift after $n$ equilibration steps (eq. S3)

$\Delta \delta^{h} E_{H S}=n \times \varepsilon \times f_{H S}$

with

$f_{H S}=\left(\frac{c_{0}-c_{H S}}{c_{0}}\right)^{1 / n}$ 
Enrichment factor $\varepsilon$ can be determined as the slope of the linear regression of eq. S3.

\section{Comparison of Rayleigh Data Evaluation with the Stepwise Partitioning Approach}

Table S2. Comparison of data evaluation, i.e., determination of enrichment factor $\varepsilon$, for benzene, triethylamine (TEA), and trichloromethane $\left(\mathrm{CHCl}_{3}\right)$ using Rayleigh equation (eq. 8) and the stepwise partitioning equation (eq. S1).

\begin{tabular}{l|cc} 
& $\begin{array}{c}\boldsymbol{\varepsilon}[\% \mathbf{\%}] \\
\text { Rayleigh equation }\end{array}$ & $\begin{array}{c}\boldsymbol{\varepsilon}[\% \text { \%] } \\
\text { stepwise partitioning }\end{array}$ \\
\hline benzene, $\boldsymbol{\varepsilon}_{\mathbf{c}}$ & $-0.12 \pm 0.03\left(\mathrm{R}^{2}=0.62\right)$ & $-0.11 \pm 0.06\left(\mathrm{R}^{2}=0.25\right)$ \\
$\mathbf{T E A}, \boldsymbol{\varepsilon}_{\mathbf{c}}$ & $0.49 \pm 0.10\left(\mathrm{R}^{2}=0.72\right)$ & $0.79 \pm 0.18\left(\mathrm{R}^{2}=0.71\right)$ \\
$\mathbf{C H C l}_{3}, \boldsymbol{\varepsilon}_{\mathbf{c}}$ & $-0.35 \pm 0.14\left(\mathrm{R}^{2}=0.38\right)$ & $-0.77 \pm 0.61\left(\mathrm{R}^{2}=0.14\right)$ \\
$\mathbf{C H C l}_{3}, \boldsymbol{\varepsilon}_{\mathbf{H}}$ & $1.79 \pm 0.25\left(\mathrm{R}^{2}=0.80\right)$ & $1.11 \pm 0.56\left(\mathrm{R}^{2}=0.35\right)$
\end{tabular}


Table S3. Isotope effects ( $\varepsilon, \%$ o) on benzene vaporization from aqueous solution obtained from calculations performed with PCM implicit solvent model.

\begin{tabular}{|c|c|c|}
\hline Method/Functional & Basis set & $\varepsilon$ \\
\hline B3LYP & $6-311++\mathrm{G}(2 \mathrm{~d}, 2 \mathrm{p})$ & 0.18 \\
\hline B3LYP & 6-31G(d,p) & 0.24 \\
\hline M06-2X & 6-31G(d,p) & 0.18 \\
\hline MP2 & $6-311++G(2 d, 2 p)$ & 0.20 \\
\hline MP2 & $6-31 G(d, p)$ & 0.27 \\
\hline B3LYP & $6-311+G(2 d f, 2 p)$ & 0.22 \\
\hline M06-2X & $6-31+G(d, p)$ & 0.43 \\
\hline $\mathrm{HF}$ & $6-311+G(2 d f, 2 p)$ & 0.24 \\
\hline $\mathrm{HF}$ & $6-31+G(d, p)$ & 0.47 \\
\hline MP2 & $6-311+G(2 d f, 2 p)$ & 0.23 \\
\hline MP2 & $6-31+G(d, p)$ & 0.44 \\
\hline B3LYP & $6-31+G(2 d f, 2 p)$ & 0.38 \\
\hline B3LYP & $6-311+G(2 d f, 2 p)$ & 0.19 \\
\hline B3LYP & $6-311+G(d, p)$ & 0.32 \\
\hline B3LYP & $6-311++G(d, p)$ & $\mathbf{0 . 1 7}$ \\
\hline M06-2X & $6-311+G(2 d f, 2 p)$ & 0.46 \\
\hline M06-2X & $6-311+G(d, p)$ & 0.47 \\
\hline $\mathrm{HF}$ & $6-31+G(2 d f, 2 p)$ & $\mathbf{0 . 3 9}$ \\
\hline $\mathrm{HF}$ & $6-311+G(2 d f, 2 p)$ & 0.16 \\
\hline $\mathrm{HF}$ & $6-311+G(d, p)$ & 0.47 \\
\hline MP2 & $6-31+G(2 d f, 2 p)$ & $\mathbf{0 . 3 9}$ \\
\hline MP2 & $6-311+G(2 d f, 2 p)$ & -0.01 \\
\hline MP2 & $6-311+G(d, p)$ & 0.27 \\
\hline B3LYP & $6-311+G(2 d f, 2 p)$ & 0.22 \\
\hline B3LYP & $6-31+G(d, p)$ & $\mathbf{0 . 3 7}$ \\
\hline $\mathrm{HF}$ & $6-311+G(2 d f, 2 p)$ & 0.24 \\
\hline $\mathrm{HF}$ & $6-31+\mathrm{G}(\mathrm{d}, \mathrm{p}$ & 0.47 \\
\hline MP2 & $6-311+G(2 d f, 2 p)$ & 0.23 \\
\hline MP2 & $6-31+G(d, p)$ & 0.41 \\
\hline B2PLYPD3 & $6-311+G(d, p)$ & $\mathbf{0 . 3 3}$ \\
\hline B2PLYPD3 & aug-cc-pvdz & 0.32 \\
\hline B2PLYPD3 & $6-311+G(2 d f, 2 p)$ & 0.27 \\
\hline B2PLYPD3 & $6-311++\mathrm{G}(2 \mathrm{df}, 2 \mathrm{p})$ & 0.23 \\
\hline B2PLYPD3 & $6-31+G(d, p)$ & 0.36 \\
\hline M06-2X & $6-311+G(d, p)$ & 0.47 \\
\hline M06-2X & aug-cc-pvTZ & 0.15 \\
\hline M06-2X & $6-311+G(2 d f, 2 p)$ & 0.28 \\
\hline
\end{tabular}




\begin{tabular}{|l|l|l|}
\hline M06-2X & $6-31+G(d, p)$ & $\mathbf{0 . 4 6}$ \\
\hline M11 & aug-cc-pvtz & $\mathbf{0 . 0 1}$ \\
\hline M11 & $6-311+G(2 d f, 2 p)$ & $\mathbf{0 . 3 2}$ \\
\hline M11 & $6-311++G(2 d f, 2 p)$ & $\mathbf{0 . 2 0}$ \\
\hline
\end{tabular}

Table S4. Isotope effects $(\varepsilon, \%)$ on trichloromethane vaporization from aqueous solution obtained from calculations performed with PCM implicit solvent model.

\begin{tabular}{|l|l|l|}
\hline Method/Functional & Basis set & $\boldsymbol{\varepsilon}$ \\
\hline B3LYP & $6-311++\mathrm{G}(2 \mathrm{~d}, 2 \mathrm{p})$ & $\mathbf{3 . 8 5}$ \\
\hline B3LYP & $6-31 \mathrm{G}(\mathrm{d}, \mathrm{p})$ & $\mathbf{3 . 7 9}$ \\
\hline MP2 & $6-311++\mathrm{G}(2 \mathrm{~d}, 2 \mathrm{p})$ & $\mathbf{3 . 1 4}$ \\
\hline B3LYP & $6-31+\mathrm{G}(2 \mathrm{df}, 2 \mathrm{p})$ & $\mathbf{3 . 7 6}$ \\
\hline B3LYP & $6-311+\mathrm{G}(\mathrm{d}, \mathrm{p})$ & $\mathbf{3 . 9 5}$ \\
\hline B3LYP & $6-311++\mathrm{G}(\mathrm{d}, \mathrm{p})$ & $\mathbf{4 . 0 2}$ \\
\hline HF & $6-31+\mathrm{G}(2 \mathrm{df}, 2 \mathrm{p})$ & $\mathbf{3 . 5 8}$ \\
\hline HF & $6-311++\mathrm{G}(\mathrm{d}, \mathrm{p})$ & $\mathbf{3 . 8 1}$ \\
\hline MP2 & $6-311+\mathrm{G}(2 \mathrm{df}, 2 \mathrm{p})$ & $\mathbf{2 . 9 0}$ \\
\hline MP2 & $6-311++\mathrm{G}(\mathrm{d}, \mathrm{p})$ & $\mathbf{3 . 0 7}$ \\
\hline B3LYP & $6-311+\mathrm{G}(2 \mathrm{df}, 2 \mathrm{p})$ & $\mathbf{3 . 5 9}$ \\
\hline B3LYP & $6-31+\mathrm{G}(\mathrm{d}, \mathrm{p})$ & $\mathbf{3 . 8 3}$ \\
\hline MP2 & $6-31+\mathrm{G}(\mathrm{d}, \mathrm{p})$ & $\mathbf{3 . 0 1}$ \\
\hline B3LYP & $6-311+\mathrm{G}(2 \mathrm{df}, 2 \mathrm{p})$ & $\mathbf{3 . 6 0}$ \\
\hline B3LYP & $6-31+\mathrm{G}(\mathrm{d}, \mathrm{p})$ & $\mathbf{3 . 8 9}$ \\
\hline MP2 & $6-311+\mathrm{G}(2 \mathrm{df}, 2 \mathrm{p})$ & $\mathbf{2 . 9 6}$ \\
\hline B2PLYP-D3 & $6-311+\mathrm{G}(\mathrm{d}, \mathrm{p})$ & $\mathbf{3 . 5 8}$ \\
\hline B2PLYP-D3 & aug-cc-pvdz & $\mathbf{3 . 4 6}$ \\
\hline B2PLYP-D3 & aug-cc-pvTz & $\mathbf{3 . 3 1}$ \\
\hline B2PLYP-D3 & $6-311+\mathrm{G}(2 \mathrm{df}, 2 \mathrm{p})$ & $\mathbf{3 . 3 4}$ \\
\hline B2PLYP-D3 & $6-311++\mathrm{G}(2 \mathrm{df}, 2 \mathrm{p})$ & $\mathbf{3 . 3 3}$ \\
\hline M11 & $6-311++\mathrm{G}(2 \mathrm{df}, 2 \mathrm{p})$ & $\mathbf{3 . 0 3}$ \\
\hline & & \\
\hline
\end{tabular}


Table S5. Isotope effects $(\varepsilon, \%)$ on triethylamine vaporization from aqueous solution obtained from calculations performed with PCM implicit solvent model.

\begin{tabular}{|c|c|c|}
\hline Method/Functional & Basis set & $\varepsilon$ \\
\hline M06-2X & $6-311++G(2 d, 2 p)$ & 0.37 \\
\hline M06-2X & $6-31 G(d, p)$ & 0.43 \\
\hline $\mathrm{HF}$ & $6-311++G(2 d, 2 p)$ & 0.42 \\
\hline B3LYP & $6-31+G(2 d f, 2 p)$ & 0.38 \\
\hline B3LYP & $6-311++G(2 d f, 2 p)$ & 0.37 \\
\hline B3LYP & $6-311+G(d, p)$ & 0.38 \\
\hline B3LYP & $6-311++\mathrm{G}(\mathrm{d}, \mathrm{p})$ & 0.38 \\
\hline M06-2X & $6-31+G(2 d f, 2 p)$ & 0.39 \\
\hline M06-2X & $6-311++G(2 d f, 2 p)$ & 0.37 \\
\hline M06-2X & $6-311+G(d, p)$ & 0.38 \\
\hline M06-2X & $6-311++\mathrm{G}(\mathrm{d}, \mathrm{p})$ & 0.37 \\
\hline $\mathrm{HF}$ & $6-31+G(2 d f, 2 p)$ & 0.43 \\
\hline $\mathrm{HF}$ & $6-311++G(2 d f, 2 p)$ & 0.43 \\
\hline $\mathrm{HF}$ & $6-311+G(d, p)$ & 0.44 \\
\hline $\mathrm{HF}$ & $6-311++\mathrm{G}(\mathrm{d}, \mathrm{p})$ & 0.43 \\
\hline MP2 & $6-31+G(2 d f, 2 p)$ & 0.39 \\
\hline MP2 & $6-311++G(2 d f, 2 p)$ & 0.39 \\
\hline MP2 & $6-311+G(d, p)$ & 0.40 \\
\hline MP2 & $6-311++\mathrm{G}(\mathrm{d}, \mathrm{p})$ & 0.40 \\
\hline B3LYP & $6-311+G(2 d f, 2 p)$ & 0.37 \\
\hline B3LYP & $6-31+\mathrm{G}(\mathrm{d}, \mathrm{p})$ & 0.40 \\
\hline M06-2X & $6-311+G(2 d f, 2 p)$ & 0.37 \\
\hline M06-2X & $6-31+\mathrm{G}(\mathrm{d}, \mathrm{p})$ & 0.42 \\
\hline $\mathrm{HF}$ & $6-311+G(2 d f, 2 p)$ & 0.43 \\
\hline $\mathrm{HF}$ & $6-31+\mathrm{G}(\mathrm{d}, \mathrm{p})$ & 0.45 \\
\hline MP2 & $6-311+G(2 d f, 2 p)$ & 0.39 \\
\hline MP2 & $6-31+\mathrm{G}(\mathrm{d}, \mathrm{p})$ & 0.41 \\
\hline B2PLYP-D3 & $6-311+G(d, p)$ & 0.41 \\
\hline B2PLYP-D3 & aug-cc-pvdz & 0.41 \\
\hline B2PLYP-D3 & $6-311+G(2 d f, 2 p)$ & 0.40 \\
\hline B2PLYP-D3 & $6-311++G(2 d f, 2 p)$ & 0.40 \\
\hline B2PLYP-D3 & $6-31+\mathrm{G}(\mathrm{d}, \mathrm{p})$ & 0.43 \\
\hline M06-2X & $6-311+G(d, p)$ & 0.38 \\
\hline M06-2X & aug-cc-pvdz & 0.38 \\
\hline M06-2X & aug-cc-pvtz & 0.36 \\
\hline M06-2X & $6-311+G(2 d f, 2 p)$ & 0.37 \\
\hline M06-2X & $6-311++G(2 d f, 2 p)$ & 0.37 \\
\hline
\end{tabular}




\begin{tabular}{|l|l|l|}
\hline M11 & 6-311+G(d,p) & $\mathbf{0 . 3 7}$ \\
\hline M11 & aug-cc-pvdz & $\mathbf{0 . 3 8}$ \\
\hline M11 & aug-cc-pvtz & $\mathbf{0 . 3 4}$ \\
\hline M11 & 6-311+G(2df,2p) & $\mathbf{0 . 3 4}$ \\
\hline M11 & 6-311++G(2df,2p) & $\mathbf{0 . 3 4}$ \\
\hline
\end{tabular}

Table S6. Normal modes (in $\mathrm{cm}^{-1}$ ) obtained based on Hessian matrix calculation for ${ }^{12} \mathrm{C}_{6} \mathrm{H}_{6}$ and ${ }^{13} \mathrm{C}_{6} \mathrm{H}_{6}$ at the B2PLYP/6-311+G(2df,2p) level for the complex containing one water molecule (benzene-WAT) and the isolated molecule (benzene-air). Modes contributing the most to the overall isotope effect of $-0.15 \%$ are shown in a bold font. The table presents the exemplary data for one $(\mathrm{C} 1)$ out of six carbon atoms.

\begin{tabular}{|c|c|c|c|c|c|}
\hline \multicolumn{3}{|c|}{ benzene-WAT } & \multicolumn{3}{|c|}{ benzene-air } \\
\hline${ }^{12} \mathrm{Cv}$ & ${ }^{13} \mathrm{C} v$ & $\begin{array}{c}{ }^{12} \mathrm{C} v / /{ }^{13} \mathrm{C} \\
v \text { ratio }\end{array}$ & ${ }^{12} \mathrm{Cv}$ & ${ }^{13} \mathrm{C} \mathrm{V}$ & $\begin{array}{c}{ }^{12} \mathrm{C} v / /{ }^{13} \mathrm{C} v \\
\text { ratio }\end{array}$ \\
\hline $12 \mathrm{C6H6}$ & 13С6Н6 & ratio & $12 \mathrm{C6H6}$ & 13С6Н6 & ratio \\
\hline 18.8565 & 18.8098 & 1.0000 & 402.287 & 398.3914 & 0.9972 \\
\hline 38.7018 & 38.6851 & 1.0000 & 402.3638 & 402.3603 & 1.0000 \\
\hline 44.723 & 44.5051 & 1.0000 & 620.1915 & 613.6334 & 0.9932 \\
\hline 97.8231 & 97.7222 & 1.0000 & 620.1955 & 618.9849 & 0.9987 \\
\hline 127.7616 & 127.7462 & 1.0000 & 627.0078 & 623.2375 & 0.9960 \\
\hline 248.2997 & 248.2977 & 1.0000 & 685.9679 & 685.6396 & 0.9996 \\
\hline 400.4271 & 396.572 & 1.0028 & 857.7253 & 855.3757 & 0.9969 \\
\hline 403.1982 & 403.1976 & 1.0000 & 857.7336 & 857.7333 & 1.0000 \\
\hline 619.3238 & 612.7792 & 1.0068 & 959.9548 & 956.2065 & 0.9948 \\
\hline 619.7902 & 618.5795 & 1.0013 & 959.9756 & 959.9756 & 1.0000 \\
\hline 641.4977 & 637.7251 & 1.0040 & 960.1472 & 960.1235 & 1.0000 \\
\hline 692.9 & 692.5478 & 1.0004 & 1017.1815 & 1005.6085 & 0.9834 \\
\hline 864.6652 & 862.2553 & 1.0032 & 1020.462 & 1019.006 & 0.9979 \\
\hline 866.4841 & 866.4836 & 1.0000 & 1068.33 & 1065.037 & 0.9951 \\
\hline 969.2854 & 965.8833 & 1.0048 & 1068.352 & 1066.035 & 0.9966 \\
\hline 969.5207 & 969.296 & 1.0003 & 1186.357 & 1185.774 & 0.9991 \\
\hline 978.5597 & 978.2452 & 1.0004 & 1209.018 & 1206.896 & 0.9966 \\
\hline 1015.9176 & 1005.0124 & 1.0159 & 1209.034 & 1209.022 & 1.0000 \\
\hline 1020.968 & 1018.89 & 1.0030 & 1381.3986 & 1371.0274 & 0.9827 \\
\hline 1067.388 & 1064.88 & 1.0037 & 1388.89 & 1388.022 & 0.9985 \\
\hline 1068.228 & 1065.085 & 1.0047 & 1525.614 & 1517.731 & 0.9863 \\
\hline 1187.514 & 1186.908 & 1.0010 & 1525.636 & 1522.631 & 0.9948 \\
\hline 1209.337 & 1207.774 & 1.0025 & 1653.0157 & 1638.9667 & 0.9751 \\
\hline 1209.93 & 1209.333 & 1.0009 & 1653.034 & 1649.928 & 0.9944 \\
\hline 1380.079 & 1369.8279 & 1.0173 & 3208.454 & 3205.632 & 0.9941 \\
\hline 1389.298 & 1388.318 & 1.0017 & 3218.165 & 3214.826 & 0.9931 \\
\hline 1524.47 & 1516.635 & 1.0138 & 3218.193 & 3218.186 & 1.0000 \\
\hline
\end{tabular}




\begin{tabular}{|c|c|c|c|c|c|}
\hline 1525.076 & 1522.09 & 1.0052 & 3233.576 & 3230.562 & 0.9937 \\
\hline 1629.572 & 1629.555 & 1.0000 & 3233.605 & 3233.588 & 1.0000 \\
\hline 1650.0858 & 1637.2653 & 1.0232 & 3243.209 & 3242.037 & 0.9976 \\
\hline 1651.274 & 1646.988 & 1.0077 & & & \\
\hline 3213.44 & 3211.137 & 1.0048 & & & \\
\hline 3222.232 & 3220.487 & 1.0036 & & & \\
\hline 3223.972 & 3222.278 & 1.0035 & & & \\
\hline 3236.839 & 3235.377 & 1.0031 & & & \\
\hline 3238.856 & 3236.878 & 1.0041 & & & \\
\hline 3246.909 & 3245.67 & 1.0026 & & & \\
\hline 3822.484 & 3822.484 & 1.0000 & & & \\
\hline 3945.59 & 3945.59 & 1.0000 & & & \\
\hline
\end{tabular}


Table S7a. Carbon isotope effects $(\varepsilon, \%)$ on water-air equilibrium partitioning obtained for the individual positions using the DFT/TIP3P models of benzene (BEN), chloroform (TCM), and triethylamine (TEA) aqueous solution (only the molecule of the respective solute was treated quantum mechanically) presented as a mean value with standard deviation.

\begin{tabular}{|c|c|c|c|c|c|c|c|}
\hline & \multicolumn{2}{|c|}{ BEN } & \multicolumn{2}{|c|}{ TCM } & \multicolumn{3}{|c|}{ TEA } \\
\hline & $\begin{array}{c}\text { Mean } \\
\text { 3N-6/3N }\end{array}$ & $\begin{array}{l}\text { Std.dev. } \\
\text { 3N-6/3N }\end{array}$ & $\begin{array}{c}\text { Mean } \\
\text { 3N-6/3N }\end{array}$ & $\begin{array}{l}\text { Std.dev. } \\
\text { 3N-6/3N }\end{array}$ & $\begin{array}{c}\text { Mean } \\
\text { 3N-6/3N }\end{array}$ & $\begin{array}{l}\text { Std.dev. } \\
\text { 3N-6/3N }\end{array}$ & \\
\hline C1 & $0.45 / 0.20$ & $0.62 / 0.54$ & $0.08 / 0.04$ & $0.46 / 0.47$ & $-0.10 /-0.18$ & $0.74 / 0.74$ & \\
\hline $\mathrm{C2}$ & $0.37 / 0.13$ & $0.79 / 0.73$ & & & $-0.49 /-0.59$ & $0.24 / 0.23$ & \\
\hline $\mathrm{C3}$ & $0.55 / 0.32$ & $0.67 / 0.62$ & & & $-1.44 /-1.57$ & $1.34 / 1.35$ & \\
\hline C4 & $0.75 / 0.44$ & $0.59 / 0.55$ & & & $-0.45 /-0.63$ & $0.69 / 0.66$ & \\
\hline C5 & $0.93 / 0.58$ & $0.44 / 0.54$ & & & $-0.23 /-0.32$ & $0.66 / 0.67$ & \\
\hline C6 & $0.62 / 0.32$ & $0.35 / 0.37$ & & & $-0.05 /-0.25$ & $0.44 / 0.42$ & \\
\hline
\end{tabular}

Table S7b. Carbon isotope effects $(\varepsilon, \%)$ on water-air equilibrium partitioning obtained for the individual positions using the DFT/TIP3P models of benzene (BEN), chloroform (TCM), and triethylamine (TEA) aqueous solution (the solute molecule and the water molecule forming a hydrogen bond with it were included in the QM part) presented as a mean value with standard deviation.

\begin{tabular}{|c|c|c|c|c|c|c|c|}
\hline & \multicolumn{2}{|c|}{ BEN } & \multicolumn{2}{|c|}{ TCM } & \multicolumn{3}{|c|}{ TEA } \\
\hline & $\begin{array}{c}\text { Mean } \\
\text { 3N-6/3N }\end{array}$ & $\begin{array}{c}\text { Std.dev. } \\
\text { 3N- } \\
\text { 6/3N }\end{array}$ & $\begin{array}{c}\text { Mean } \\
\text { 3N-6/3N }\end{array}$ & $\begin{array}{l}\text { Std.dev. } \\
\text { 3N-6/3N }\end{array}$ & $\begin{array}{c}\text { Mean } \\
\text { 3N-6/3N }\end{array}$ & $\begin{array}{l}\text { Std.dev. } \\
\text { 3N-6/3N }\end{array}$ & \\
\hline C1 & $-0.23 /-0.36$ & $0.35 / 0.37$ & $0.34 / 0.31$ & $0.27 / 0.28$ & $-0.08 /-0.15$ & $0.48 /$ & \\
\hline $\mathrm{C2}$ & $-0.25 /-0.37$ & $0.41 / 0.45$ & & & $-0.28 /-0.37$ & $0.25 / 0.25$ & \\
\hline $\mathbf{C 3}$ & $-0.21 /-0.32$ & $0.53 / 0.58$ & & & $-1.68 /-1.79$ & $1.17 / 1.17$ & \\
\hline C4 & $-0.09 /-0.20$ & $0.49 / 0.51$ & & & $-0.57 /-0.70$ & $0.50 / 0.52$ & \\
\hline C5 & $-0.07 /-0.19$ & $0.55 / 0.58$ & & & $-0.07 /-0.16$ & $0.63 / 0.64$ & \\
\hline C6 & $-0.22 /-0.35$ & \begin{tabular}{|l|l}
$0.50 / 0.53$ \\
\end{tabular} & & & $-0.27 /-0.44$ & $0.46 / 0.44$ & \\
\hline
\end{tabular}

DFT for benzene - B2PLYP-D3/6-31+G(d,p), for TCM and TEA - B3LYP/6-31+G(d,p). 
Table S8. Benzene-water interaction energy and its components computed by SAPT for microsolvation solvent models prepared manually, obtained at the B2PLYP-D3/6-311+G(2df,2p) theory level. Absolute interaction energies are given in $\mathrm{kcal} \cdot \mathrm{mol}^{-1}$.

\begin{tabular}{|c|c|c|c|c|c|c|c|c|c|}
\hline \multirow[t]{2}{*}{$\begin{array}{l}\text { Total number of } \\
\text { water molecules }\end{array}$} & \multirow[t]{2}{*}{$\begin{array}{c}\text { No. of } \\
\text { water mol. }\end{array}$} & \multicolumn{4}{|c|}{$\begin{array}{c}\text { Absolute interaction energy } \\
\text { contributions }\end{array}$} & \multicolumn{3}{|c|}{$\begin{array}{c}\text { Ratios to } \\
\text { electrostatics }\end{array}$} & \multirow{2}{*}{$\begin{array}{c}\text { Total SAPT } \\
\text { energy }\end{array}$} \\
\hline & & $\begin{array}{c}\begin{array}{c}\text { Electrost } \\
\text { atics }\end{array} \\
\end{array}$ & \begin{tabular}{|c} 
Excha \\
nge
\end{tabular} & $\begin{array}{c}\text { Induc } \\
\text { tion }\end{array}$ & $\begin{array}{l}\text { Disper } \\
\text { sion }\end{array}$ & $\begin{array}{c}\text { Excha } \\
\text { nge }\end{array}$ & $\begin{array}{c}\text { Induc } \\
\text { tion }\end{array}$ & $\begin{array}{c}\text { Disper } \\
\text { sion }\end{array}$ & \\
\hline 1 & 1 & -2.98 & 3.89 & -1.06 & -3.17 & -1.31 & 0.36 & 1.07 & -3.33 \\
\hline 2 & $\begin{array}{c}\text { Total } \\
\text { interaction }\end{array}$ & -5.75 & 7.31 & -2.08 & -6.12 & & & & -6.64 \\
\hline \multirow{2}{*}{$\begin{array}{l}\text { Per each water } \\
\text { molecule present in a } \\
\text { cluster computed } \\
\text { separately } \\
\end{array}$} & 1 & -2.87 & 3.65 & -1.04 & -3.06 & -1.27 & 0.36 & 1.06 & -3.32 \\
\hline & 2 & -2.88 & 3.66 & -1.04 & -3.06 & -1.27 & 0.36 & 1.06 & -3.32 \\
\hline 4 & $\begin{array}{c}\text { Total } \\
\text { interaction }\end{array}$ & -9.66 & 13.26 & -3.09 & -9.93 & & & & -9.42 \\
\hline \multirow{4}{*}{$\begin{array}{l}\text { Per each water } \\
\text { molecule present in a } \\
\text { cluster computed } \\
\text { separately }\end{array}$} & 1 & -2.89 & 3.71 & -1.00 & -3.14 & -1.28 & 0.34 & 1.09 & -3.32 \\
\hline & 2 & -0.70 & 0.36 & -0.07 & -0.71 & -0.51 & 0.10 & 1.01 & -1.13 \\
\hline & 3 & -2.65 & 3.68 & -0.46 & -2.42 & -1.39 & 0.17 & 0.92 & -1.86 \\
\hline & 4 & -3.42 & 5.51 & -1.55 & -3.66 & -1.61 & 0.45 & 1.07 & -3.12 \\
\hline 6 & $\begin{array}{c}\text { Total } \\
\text { interaction }\end{array}$ & -7.95 & 12.24 & -2.08 & -10.32 & & & & -8.11 \\
\hline \multirow{6}{*}{$\begin{array}{l}\text { Per each water } \\
\text { molecule present in a } \\
\text { cluster computed } \\
\text { separately }\end{array}$} & 1 & -0.84 & 0.50 & -0.14 & -0.89 & -0.59 & 0.16 & 1.06 & -1.37 \\
\hline & 2 & -1.55 & 2.59 & -0.23 & -1.97 & -1.67 & 0.15 & 1.27 & -1.16 \\
\hline & 3 & -0.79 & 0.61 & -0.13 & -0.79 & -0.77 & 0.17 & 1.01 & -1.10 \\
\hline & 4 & 0.15 & 0.34 & -0.12 & -0.73 & 2.25 & -0.81 & -4.82 & -0.36 \\
\hline & 5 & -3.39 & 5.66 & -1.23 & -3.99 & -1.67 & 0.36 & 1.18 & -2.94 \\
\hline & 6 & -1.54 & 2.55 & -0.23 & -1.96 & -1.65 & 0.15 & 1.27 & -1.18 \\
\hline $6_{o p t}$ & $\begin{array}{c}\text { Total } \\
\text { interaction }\end{array}$ & -9.19 & 13.80 & -2.98 & -11.14 & & & & -9.50 \\
\hline \multirow{6}{*}{$\begin{array}{l}\text { Per each water } \\
\text { molecule present in a } \\
\text { cluster computed } \\
\text { separately }\end{array}$} & 1 & -1.53 & 2.41 & -0.23 & -1.88 & -1.58 & 0.15 & 1.23 & -1.23 \\
\hline & 2 & -1.25 & 2.02 & -0.20 & -1.74 & -1.62 & 0.16 & 1.39 & -1.16 \\
\hline & 3 & -2.58 & 4.21 & -1.12 & -3.41 & -1.63 & 0.44 & 1.32 & -2.90 \\
\hline & 4 & 0.05 & 0.24 & -0.09 & -0.58 & 4.89 & -1.87 & -11.73 & -0.38 \\
\hline & 5 & -0.92 & 0.92 & -0.24 & -0.95 & -1.01 & 0.26 & 1.04 & -1.19 \\
\hline & 6 & -2.96 & 3.99 & -1.10 & -2.58 & -1.35 & 0.37 & 0.87 & -2.65 \\
\hline
\end{tabular}

opt-geometry preoptimized with the PM3 method. 
Table S9. Normal modes (in $\mathrm{cm}^{-1}$ ) obtained based on Hessian matrix calculation for ${ }^{12} \mathrm{CHCl}_{3}$ and ${ }^{13} \mathrm{CHCl}_{3}$ at the B3LYP/6-311+G(2df,2p) level for the complex containing three water molecules and the isolated chloroform molecule. Modes contributing the most to the overall isotope effect of $1.4 \%$ are shown in a bold font.

\begin{tabular}{|c|c|c|c|c|c|}
\hline \multicolumn{3}{|c|}{$\mathrm{CHCl}_{3}$-WAT } & \multicolumn{3}{|c|}{$\mathrm{CHCl}_{3}$-air } \\
\hline${ }^{12} \mathrm{C} \mathrm{V}$ & ${ }^{13} \mathrm{C} \mathrm{V}$ & $\begin{array}{c}{ }^{12} \mathrm{C} v /{ }^{13} \mathrm{C} v \\
\text { ratio }\end{array}$ & ${ }^{12} \mathrm{CV}$ & ${ }^{13} \mathrm{C} \mathrm{v}$ & $\begin{array}{c}{ }^{12} \mathrm{C} v / /{ }^{13} \mathrm{Cv} \\
\text { ratio }\end{array}$ \\
\hline 9.900 & 9.899 & 1.0000 & 256.670 & 256.239 & 0.9998 \\
\hline 36.832 & 36.803 & 1.0000 & 256.682 & 256.252 & 0.9998 \\
\hline 43.431 & 43.427 & 1.0000 & 362.547 & 360.466 & 0.9986 \\
\hline 51.563 & 51.526 & 1.0000 & 663.049 & 645.252 & 0.9808 \\
\hline 59.617 & 59.559 & 1.0000 & 730.574 & 707.826 & 0.9738 \\
\hline 69.390 & 69.281 & 1.0000 & 730.674 & 707.921 & 0.9738 \\
\hline 137.434 & 137.434 & 1.0000 & 1237.195 & 1230.304 & 0.9890 \\
\hline 140.800 & 140.774 & 1.0000 & 1237.218 & 1230.326 & 0.9890 \\
\hline 152.480 & 152.478 & 1.0000 & 3187.699 & 3166.408 & 0.9566 \\
\hline 162.893 & 162.890 & 1.0000 & & & \\
\hline 188.162 & 188.162 & 1.0000 & & & \\
\hline 196.767 & 196.766 & 1.0000 & & & \\
\hline 223.784 & 223.783 & 1.0000 & & & \\
\hline 254.552 & 254.101 & 1.0002 & & & \\
\hline 256.391 & 255.994 & 1.0002 & & & \\
\hline 288.689 & 288.685 & 1.0000 & & & \\
\hline 330.663 & 330.463 & 1.0001 & & & \\
\hline 366.788 & 365.045 & 1.0012 & & & \\
\hline 517.660 & 517.608 & 1.0000 & & & \\
\hline 651.260 & 633.384 & 1.0193 & & & \\
\hline 680.095 & 668.486 & 1.0130 & & & \\
\hline 682.064 & 677.948 & 1.0046 & & & \\
\hline 720.336 & 711.789 & 1.0100 & & & \\
\hline 737.404 & 716.749 & 1.0245 & & & \\
\hline 1304.407 & 1297.227 & 1.0118 & & & \\
\hline 1329.702 & 1322.742 & 1.0116 & & & \\
\hline 1632.366 & 1632.366 & 1.0000 & & & \\
\hline 1632.801 & 1632.800 & 1.0000 & & & \\
\hline 1658.277 & 1658.275 & 1.0000 & & & \\
\hline 3077.117 & 3056.159 & 1.0444 & & & \\
\hline 3670.870 & 3670.853 & 1.0000 & & & 6 \\
\hline 3744.275 & 3744.275 & 1.0000 & & & \\
\hline 3789.833 & 3789.833 & 1.0000 & & & \\
\hline 3790.907 & 3790.907 & 1.0000 & & & \\
\hline 3897.752 & 3897.752 & 1.0000 & & & \\
\hline 3898.453 & 3898.453 & 1.0000 & & & \\
\hline
\end{tabular}


Table S10. Trichloromethane-water interaction energy and its components computed by SAPT for solvent models prepared manually and by cutting out solvent molecules from water box on microsolvation model structures obtained in calculations with the B3LYP DFT functional with smaller and larger basis set. $6-31+\mathrm{G}(\mathrm{d}, \mathrm{p})$ and $6-311+\mathrm{G}(2 \mathrm{df}, 2 \mathrm{p})$, respectively. Absolute interaction energies are given in $\mathrm{kcal} \cdot \mathrm{mol}^{-1}$.

\begin{tabular}{|c|c|c|c|c|c|c|c|c|c|}
\hline \multirow{2}{*}{$\begin{array}{c}\text { Total number } \\
\text { of water } \\
\text { molecules }\end{array}$} & \multirow{2}{*}{$\begin{array}{c}\text { No. of } \\
\text { water } \\
\text { mol. }\end{array}$} & \multicolumn{4}{|c|}{ Absolute interaction energy contributions } & \multicolumn{3}{|c|}{ Ratios to electrostatics } & \multirow{2}{*}{$\begin{array}{c}\text { Total SAPT } \\
\text { energy }\end{array}$} \\
\hline & & Electrostatics & Exchange & Induction & Dispersion & Exchange & Induction & Dispersion & \\
\hline \multicolumn{10}{|c|}{ Solvent models prepared manually } \\
\hline \multicolumn{10}{|c|}{ 6-31+G(d,p) } \\
\hline 4 opt & $\begin{array}{c}\text { Total } \\
\text { interaction }\end{array}$ & -8.60 & 9.53 & -2.61 & -4.56 & & & & -6.24 \\
\hline \multirow{4}{*}{$\begin{array}{l}\text { Per each water } \\
\text { molecule present in } \\
\text { a cluster computed } \\
\text { separately }\end{array}$} & 1 & -6.86 & 7.68 & -1.95 & -3.03 & -1.12 & 0.28 & 0.44 & -4.16 \\
\hline & 2 & -0.11 & 0.00 & -0.01 & -0.05 & 0.00 & 0.06 & 0.42 & -0.16 \\
\hline & 3 & 0.07 & 0.00 & -0.01 & -0.05 & 0.01 & -0.09 & -0.73 & 0.01 \\
\hline & 4 & -1.70 & 1.85 & -0.64 & -1.44 & -1.09 & 0.38 & 0.85 & -1.93 \\
\hline \multicolumn{10}{|c|}{ Solvent models prepared by cutting out solvent molecules from water box } \\
\hline 5 & $\begin{array}{c}\text { Total } \\
\text { interaction }\end{array}$ & -11.90 & 14.68 & -3.88 & -6.22 & -1.23 & 0.33 & 0.52 & -7.32 \\
\hline \multirow{5}{*}{$\begin{array}{l}\text { Per each water } \\
\text { molecule present in } \\
\text { a cluster computed } \\
\text { separately }\end{array}$} & 1 & -0.55 & 0.01 & -0.03 & -0.22 & -0.02 & 0.05 & 0.40 & -0.79 \\
\hline & 2 & -2.43 & 3.99 & -1.23 & -2.28 & -1.64 & 0.51 & 0.94 & -1.94 \\
\hline & 3 & -0.06 & 0.00 & 0.00 & -0.05 & -0.01 & 0.09 & 0.93 & -0.11 \\
\hline & 4 & -8.31 & 10.68 & -2.60 & -3.55 & -1.28 & 0.31 & 0.43 & -3.78 \\
\hline & 5 & -0.55 & 0.00 & -0.02 & -0.12 & -0.01 & 0.04 & 0.22 & -0.69 \\
\hline 7 & $\begin{array}{c}\text { Total } \\
\text { interaction }\end{array}$ & -8.70 & 10.09 & -2.64 & -5.09 & -1.16 & 0.30 & 0.58 & -6.34 \\
\hline \multirow{7}{*}{$\begin{array}{l}\text { Per each water } \\
\text { molecule present in } \\
\text { a cluster computed } \\
\text { separately }\end{array}$} & 1 & 0.02 & 0.00 & 0.00 & -0.03 & 0.00 & -0.27 & -1.72 & -0.02 \\
\hline & 2 & -2.09 & 2.43 & -0.79 & -1.71 & -1.16 & 0.38 & 0.82 & -2.15 \\
\hline & 3 & -0.33 & 0.00 & -0.02 & -0.11 & -0.01 & 0.06 & 0.32 & -0.45 \\
\hline & 4 & -0.15 & 0.00 & -0.01 & -0.08 & -0.01 & 0.07 & 0.56 & -0.24 \\
\hline & 5 & -6.71 & 7.66 & -1.80 & -3.05 & -1.14 & 0.27 & 0.45 & -3.90 \\
\hline & 6 & 0.15 & 0.00 & 0.00 & -0.04 & 0.00 & -0.03 & -0.24 & 0.11 \\
\hline & 7 & 0.42 & 0.00 & -0.02 & -0.08 & 0.00 & -0.04 & -0.19 & 0.32 \\
\hline 8 & $\begin{array}{c}\text { Total } \\
\text { interaction }\end{array}$ & -10.11 & 11.82 & -3.11 & -6.86 & -1.17 & 0.31 & 0.68 & -8.27 \\
\hline \multirow{4}{*}{$\begin{array}{l}\text { Per each water } \\
\text { molecule present in } \\
\text { a cluster computed } \\
\text { separately }\end{array}$} & 1 & -7.35 & 8.69 & -2.11 & -3.20 & -1.18 & 0.29 & 0.44 & -3.97 \\
\hline & 2 & -0.55 & 0.01 & -0.03 & -0.15 & -0.01 & 0.05 & 0.27 & -0.71 \\
\hline & 3 & -1.27 & 1.74 & -0.45 & -1.81 & -1.37 & 0.35 & 1.42 & -1.79 \\
\hline & 4 & 0.69 & 0.02 & -0.04 & -0.22 & 0.02 & -0.06 & -0.32 & 0.44 \\
\hline
\end{tabular}




\begin{tabular}{|c|c|c|c|c|c|c|c|c|c|}
\hline & 5 & -1.65 & 1.36 & -0.46 & -1.29 & -0.82 & 0.28 & 0.78 & -2.03 \\
\hline & 6 & 0.14 & 0.01 & -0.02 & -0.13 & 0.06 & -0.14 & -0.93 & 0.00 \\
\hline & 7 & 0.01 & 0.00 & 0.00 & -0.04 & 0.00 & -0.53 & -4.24 & -0.03 \\
\hline & 8 & -0.14 & 0.00 & 0.00 & -0.03 & 0.00 & 0.03 & 0.24 & -0.17 \\
\hline \multicolumn{10}{|c|}{$6-311+G(2 d f, 2 p)$} \\
\hline & & & & & & & & & \\
\hline 5 & $\begin{array}{c}\text { Total } \\
\text { interaction }\end{array}$ & -11.57 & 14.07 & -3.65 & -6.33 & -1.22 & 0.32 & 0.55 & -7.47 \\
\hline \multirow{5}{*}{$\begin{array}{l}\text { Per each water } \\
\text { molecule present in } \\
\text { a cluster computed } \\
\text { separately }\end{array}$} & 1 & -0.54 & 0.02 & -0.03 & -0.24 & -0.03 & 0.06 & 0.44 & -0.79 \\
\hline & 2 & -2.68 & 4.54 & -1.29 & -2.58 & -1.69 & 0.48 & 0.96 & -2.00 \\
\hline & 3 & -0.05 & 0.00 & -0.01 & -0.06 & -0.01 & 0.10 & 1.16 & -0.12 \\
\hline & 4 & -7.75 & 9.51 & -2.30 & -3.31 & -1.23 & 0.30 & 0.43 & -3.85 \\
\hline & 5 & -0.55 & 0.01 & -0.02 & -0.14 & -0.01 & 0.04 & 0.26 & -0.70 \\
\hline 7 & \begin{tabular}{c|} 
Total \\
interaction \\
\end{tabular} & -12.91 & 16.00 & -4.26 & -7.55 & -1.24 & 0.33 & 0.58 & -8.73 \\
\hline \multirow{7}{*}{$\begin{array}{l}\text { Per each water } \\
\text { molecule present in } \\
\text { a cluster computed } \\
\text { separately }\end{array}$} & 1 & -0.72 & 0.03 & -0.04 & -0.30 & -0.05 & 0.06 & 0.42 & -1.02 \\
\hline & 2 & -2.39 & 4.31 & -1.28 & -2.37 & -1.80 & 0.53 & 0.99 & -1.73 \\
\hline & 3 & -0.49 & 0.00 & -0.03 & -0.15 & -0.01 & 0.06 & 0.30 & -0.66 \\
\hline & 4 & -0.26 & 0.00 & -0.02 & -0.13 & -0.02 & 0.06 & 0.50 & -0.40 \\
\hline & 5 & 0.10 & 0.01 & -0.01 & -0.13 & 0.11 & -0.12 & -1.35 & -0.04 \\
\hline & 6 & -8.33 & 10.82 & -2.57 & -3.53 & -1.30 & 0.31 & 0.42 & -3.62 \\
\hline & 7 & -0.83 & 0.82 & -0.32 & -0.94 & -0.99 & 0.39 & 1.14 & -1.27 \\
\hline 10 & \begin{tabular}{c|c|} 
Total \\
interaction \\
\end{tabular} & -6.46 & 6.66 & -1.81 & -4.60 & -1.03 & 0.28 & 0.71 & -6.21 \\
\hline \multirow{10}{*}{$\begin{array}{l}\text { Per each water } \\
\text { molecule present in } \\
\text { a cluster computed } \\
\text { separately }\end{array}$} & 1 & -5.63 & 5.75 & -1.50 & -2.58 & -1.02 & 0.27 & 0.46 & -3.96 \\
\hline & 2 & 0.84 & 0.24 & -0.07 & -0.59 & 0.29 & -0.08 & -0.71 & 0.42 \\
\hline & 3 & -0.51 & 0.60 & -0.17 & -0.85 & -1.18 & 0.33 & 1.67 & -0.92 \\
\hline & 4 & 0.05 & 0.00 & 0.00 & -0.02 & 0.00 & -0.07 & -0.44 & 0.02 \\
\hline & 5 & -0.65 & 0.06 & -0.03 & -0.31 & -0.09 & 0.05 & 0.48 & -0.93 \\
\hline & 6 & -0.13 & 0.00 & 0.00 & -0.02 & 0.00 & 0.02 & 0.12 & -0.15 \\
\hline & 7 & -0.09 & 0.00 & -0.01 & -0.11 & -0.02 & 0.13 & 1.15 & -0.21 \\
\hline & 8 & 0.17 & 0.00 & 0.00 & -0.03 & 0.00 & -0.03 & -0.17 & 0.14 \\
\hline & 9 & -0.49 & 0.00 & -0.02 & -0.08 & 0.00 & 0.04 & 0.16 & -0.59 \\
\hline & 10 & -0.01 & 0.00 & 0.00 & -0.01 & 0.00 & 0.20 & 1.61 & -0.02 \\
\hline 12 & \begin{tabular}{c|} 
Total \\
interaction
\end{tabular} & -7.37 & 7.91 & -2.32 & -5.18 & -1.07 & 0.31 & 0.70 & -6.96 \\
\hline \multirow{4}{*}{$\begin{array}{c}\text { Per each water } \\
\text { molecule present in } \\
\text { a cluster computed } \\
\text { separately }\end{array}$} & 1 & -0.35 & 0.00 & -0.01 & -0.06 & 0.00 & 0.04 & 0.18 & -0.43 \\
\hline & 2 & -6.05 & 6.49 & -1.68 & -2.71 & -1.07 & 0.28 & 0.45 & -3.95 \\
\hline & 3 & -1.01 & 1.05 & -0.39 & -1.05 & -1.04 & 0.39 & 1.04 & -1.40 \\
\hline & 4 & 0.12 & 0.00 & 0.00 & -0.02 & 0.00 & -0.02 & -0.16 & 0.10 \\
\hline
\end{tabular}




\begin{tabular}{|c|c|c|c|c|c|c|c|c|c|}
\hline & 5 & -0.13 & 0.00 & 0.00 & -0.01 & 0.00 & 0.02 & 0.08 & -0.15 \\
\hline & 6 & -0.20 & 0.00 & -0.01 & -0.04 & 0.00 & 0.03 & 0.18 & -0.25 \\
\hline & 7 & 0.09 & 0.00 & 0.00 & -0.01 & 0.00 & -0.03 & -0.16 & 0.08 \\
\hline & 8 & 0.89 & 0.08 & -0.06 & -0.39 & 0.09 & -0.07 & -0.43 & 0.53 \\
\hline & 9 & -0.05 & 0.00 & -0.01 & -0.04 & 0.00 & 0.11 & 0.90 & -0.09 \\
\hline & 10 & -0.68 & 0.03 & -0.04 & -0.26 & -0.04 & 0.06 & 0.37 & -0.95 \\
\hline & 11 & 0.09 & 0.00 & -0.01 & -0.06 & 0.01 & -0.12 & -0.69 & 0.02 \\
\hline & 12 & -0.09 & 0.27 & -0.10 & -0.53 & -2.86 & 1.03 & 5.64 & -0.45 \\
\hline 14 & \begin{tabular}{|c|} 
Total \\
interaction
\end{tabular} & -10.23 & 12.80 & -3.64 & -7.00 & -1.25 & 0.36 & 0.68 & -8.07 \\
\hline \multirow{14}{*}{$\begin{array}{l}\text { Per each water } \\
\text { molecule present in } \\
\text { a cluster computed } \\
\text { separately }\end{array}$} & 1 & -6.73 & 7.67 & -1.96 & -2.94 & -1.14 & 0.29 & 0.44 & -3.96 \\
\hline & 2 & 0.43 & 0.01 & -0.04 & -0.20 & 0.02 & -0.09 & -0.46 & 0.20 \\
\hline & 3 & -0.70 & 0.93 & -0.30 & -0.96 & -1.33 & 0.42 & 1.37 & -1.02 \\
\hline & 4 & 0.02 & 0.00 & 0.00 & -0.01 & 0.00 & -0.03 & -0.28 & 0.02 \\
\hline & 5 & -0.47 & 0.00 & -0.02 & -0.11 & -0.01 & 0.04 & 0.24 & -0.60 \\
\hline & 6 & 0.02 & 0.00 & 0.00 & -0.01 & 0.00 & -0.05 & -0.38 & 0.01 \\
\hline & 7 & -0.11 & 0.00 & 0.00 & -0.03 & 0.00 & 0.02 & 0.25 & -0.14 \\
\hline & 8 & -0.10 & 0.00 & -0.01 & -0.06 & -0.01 & 0.08 & 0.63 & -0.17 \\
\hline & 9 & 0.04 & 0.00 & 0.00 & -0.01 & 0.00 & -0.03 & -0.34 & 0.02 \\
\hline & 10 & -0.29 & 0.01 & -0.01 & -0.15 & -0.02 & 0.05 & 0.52 & -0.44 \\
\hline & 11 & 0.06 & 0.00 & 0.00 & -0.02 & 0.00 & -0.03 & -0.26 & 0.04 \\
\hline & 12 & -2.28 & 4.17 & -1.28 & -2.24 & -1.83 & 0.56 & 0.98 & -1.63 \\
\hline & 13 & -0.08 & 0.01 & -0.01 & -0.14 & -0.11 & 0.16 & 1.67 & -0.23 \\
\hline & 14 & -0.04 & 0.01 & -0.01 & -0.12 & -0.19 & 0.29 & 3.13 & -0.16 \\
\hline
\end{tabular}

opt-geometry preoptimized with the PM3 method. 
Table S11. Isotope effects for equilibrium partitioning of triethylamine dissolved in aqueous solution obtained from calculations performed at MP2/6-311++G(d,p) theory level using PCM implicit solvent model. Input structures we taken from potential energy surface analysis. ${ }^{6}$

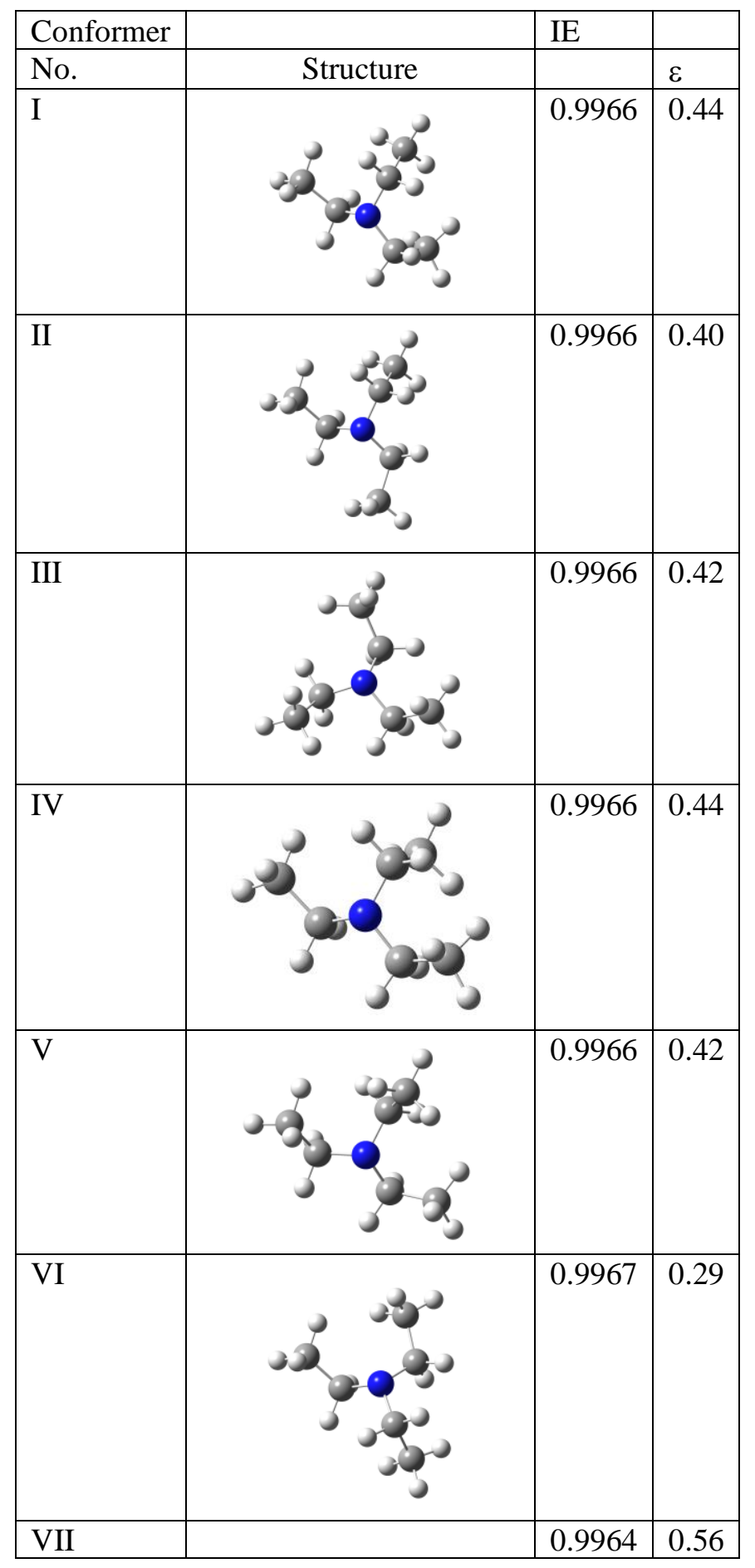


Table S12. Triethylamine-water interaction energy and its components computed by SAPT for mixed solvent models described in calculations at the B3LYP/6-31+G(d,p) theory level. Absolute interaction energies are given in $\mathrm{kcal} \cdot \mathrm{mol}^{-1}$.

\begin{tabular}{|c|c|c|c|c|c|c|c|c|c|}
\hline \multirow{2}{*}{$\begin{array}{c}\begin{array}{c}\text { Total number } \\
\text { of water } \\
\text { molecules }\end{array} \\
\text { Smaller BS } \\
\end{array}$} & \multirow[t]{2}{*}{ Water No } & \multicolumn{4}{|c|}{$\begin{array}{c}\text { Absolute interaction energy } \\
\text { contributions }\end{array}$} & \multicolumn{3}{|c|}{ Ratios to electrostatics } & \multirow{2}{*}{$\begin{array}{l}\text { Total } \\
\text { SAPT } \\
\text { energy }\end{array}$} \\
\hline & & \begin{tabular}{|c|}
$\begin{array}{c}\text { Electrostatic } \\
\mathrm{s}\end{array}$ \\
\end{tabular} & $\begin{array}{l}\text { Exch } \\
\text { ange }\end{array}$ & \begin{tabular}{|c|}
$\begin{array}{c}\text { Induc } \\
\text { tion }\end{array}$ \\
\end{tabular} & $\begin{array}{c}\text { Disper } \\
\text { sion }\end{array}$ & $\begin{array}{l}\text { Exch } \\
\text { ange }\end{array}$ & $\begin{array}{c}\text { Inducti } \\
\text { on }\end{array}$ & \begin{tabular}{|c|}
$\begin{array}{c}\text { Dispersi } \\
\text { on }\end{array}$ \\
\end{tabular} & \\
\hline 1 & 1 & -17.28 & 21.87 & -6.99 & -7.06 & -1.27 & 0.40 & 0.41 & -9.85 \\
\hline 4 & Total interaction & -17.43 & 21.85 & -7.02 & -7.26 & & & & -10.25 \\
\hline \multirow{4}{*}{$\begin{array}{l}\text { Per each water } \\
\text { molecule present in a } \\
\text { cluster computed } \\
\text { separately }\end{array}$} & 1 & -17.26 & 21.85 & -6.98 & -7.06 & -1.27 & 0.40 & 0.41 & -9.85 \\
\hline & 2 & -0.08 & 0.00 & -0.02 & -0.09 & -0.02 & 0.20 & 1.10 & -0.19 \\
\hline & 3 & 0.00 & 0.00 & 0.00 & -0.02 & 0.00 & 0.98 & 3.88 & -0.02 \\
\hline & 4 & -0.08 & 0.00 & -0.02 & -0.09 & -0.01 & 0.21 & 1.19 & -0.19 \\
\hline $\mathbf{4}_{\text {opt }}$ & Total interaction & -18.64 & 23.65 & -7.77 & -8.58 & & & & -11.76 \\
\hline \multirow{4}{*}{$\begin{array}{l}\text { Per each water } \\
\text { molecule present in a } \\
\text { cluster computed } \\
\text { separately }\end{array}$} & 1 & -18.37 & 23.36 & -7.56 & -7.12 & -1.27 & 0.41 & 0.39 & -10.09 \\
\hline & 2 & -0.85 & 0.15 & -0.08 & -0.60 & -0.18 & 0.09 & 0.71 & -1.38 \\
\hline & 3 & 0.06 & 0.06 & -0.06 & -0.41 & 0.93 & -0.99 & -6.54 & -0.35 \\
\hline & 4 & 0.51 & 0.08 & -0.06 & -0.45 & 0.16 & -0.13 & -0.90 & 0.06 \\
\hline $5_{\text {wat chk }}$ & Total interaction & -17.49 & 21.87 & -7.03 & -7.30 & & & & -10.34 \\
\hline \multirow{5}{*}{$\begin{array}{l}\text { Per each water } \\
\text { molecule present in a } \\
\text { cluster computed } \\
\text { separately }\end{array}$} & 1 & -17.27 & 21.87 & -6.99 & -7.07 & -1.27 & 0.40 & 0.41 & -9.85 \\
\hline & 2 & -0.09 & 0.00 & -0.02 & -0.10 & -0.02 & 0.23 & 1.16 & -0.21 \\
\hline & 3 & 0.00 & 0.00 & 0.00 & 0.00 & 0.00 & 0.06 & 0.27 & -0.01 \\
\hline & 4 & -0.09 & 0.00 & -0.02 & -0.13 & -0.03 & 0.26 & 1.34 & -0.24 \\
\hline & 5 & -0.03 & 0.00 & 0.00 & -0.01 & 0.00 & 0.03 & 0.30 & -0.04 \\
\hline 5opt & Total interaction & - & - & - & - & & & & - \\
\hline \multirow{5}{*}{$\begin{array}{l}\text { Per each water } \\
\text { molecule present in a } \\
\text { cluster computed } \\
\text { separately }\end{array}$} & 1 & - & - & - & - & - & - & - & - \\
\hline & 2 & -0.85 & 0.15 & -0.09 & -0.61 & -0.17 & 0.11 & 0.72 & -1.41 \\
\hline & 3 & 0.01 & 0.00 & 0.00 & -0.01 & 0.00 & -0.08 & -0.57 & $\mathbf{0 . 0 0}$ \\
\hline & 4 & 0.53 & 0.03 & -0.06 & -0.36 & 0.06 & -0.11 & -0.68 & 0.14 \\
\hline & 5 & 0.00 & 0.00 & 0.00 & 0.00 & 0.00 & 0.05 & 0.62 & $-\mathbf{0 . 0 1}$ \\
\hline \multicolumn{10}{|c|}{ Structures prepared by cutting out solvent molecules from larger system } \\
\hline 1 water & 1 & -0.14 & 0.17 & -0.12 & -0.60 & -1.21 & 0.87 & 4.34 & -0.69 \\
\hline 3 & Total interaction & -0.03 & 0.10 & -0.13 & -0.70 & & & & -0.76 \\
\hline
\end{tabular}




\begin{tabular}{|c|c|c|c|c|c|c|c|c|c|}
\hline \multirow{3}{*}{$\begin{array}{l}\text { Per each water } \\
\text { molecule present in a } \\
\text { cluster computed } \\
\text { separately }\end{array}$} & 1 & 0.02 & 0.00 & 0.00 & -0.01 & 0.00 & -0.17 & -0.88 & 0.00 \\
\hline & 2 & 0.00 & 0.04 & -0.06 & -0.29 & 15.51 & -20.97 & -100.55 & -0.30 \\
\hline & 3 & -0.05 & 0.06 & -0.07 & -0.40 & -1.10 & 1.35 & 7.61 & -0.46 \\
\hline 4 & Total interaction & -0.22 & 0.25 & -0.19 & -1.18 & & & & -1.34 \\
\hline \multirow{4}{*}{$\begin{array}{l}\text { Per each water } \\
\text { molecule present in a } \\
\text { cluster computed } \\
\text { separately }\end{array}$} & 1 & -0.08 & 0.14 & -0.07 & -0.41 & -1.68 & 0.81 & 5.13 & -0.43 \\
\hline & 2 & -0.11 & 0.06 & -0.05 & -0.30 & -0.54 & 0.51 & 2.78 & -0.40 \\
\hline & 3 & -0.02 & 0.00 & -0.01 & -0.08 & -0.07 & 0.46 & 5.47 & -0.11 \\
\hline & 4 & -0.01 & 0.05 & -0.07 & -0.38 & -4.26 & 5.27 & 29.99 & -0.40 \\
\hline 7 & Total interaction & -16.42 & 20.36 & -6.71 & -8.12 & & & & -11.21 \\
\hline \multirow{7}{*}{$\begin{array}{l}\text { Per each water } \\
\text { molecule present in a } \\
\text { cluster computed } \\
\text { separately }\end{array}$} & 1 & -0.09 & 0.14 & -0.06 & -0.44 & -1.44 & 0.68 & 4.63 & -0.46 \\
\hline & 2 & -0.09 & 0.06 & -0.05 & -0.29 & -0.63 & 0.56 & 3.31 & -0.37 \\
\hline & 3 & -0.02 & 0.00 & 0.00 & -0.01 & 0.00 & 0.07 & 0.48 & -0.03 \\
\hline & 4 & -0.19 & 0.00 & -0.01 & -0.09 & -0.01 & 0.05 & 0.47 & -0.29 \\
\hline & 5 & 0.10 & 0.04 & -0.06 & -0.35 & 0.42 & -0.60 & -3.38 & -0.26 \\
\hline & 6 & 0.54 & 0.05 & -0.04 & -0.37 & 0.08 & -0.07 & -0.68 & 0.17 \\
\hline & 7 & -16.67 & 20.08 & -6.48 & -6.57 & -1.20 & 0.39 & 0.39 & -9.96 \\
\hline
\end{tabular}

opt-geometry preoptimized with the PM3 method. 


\section{Figures}
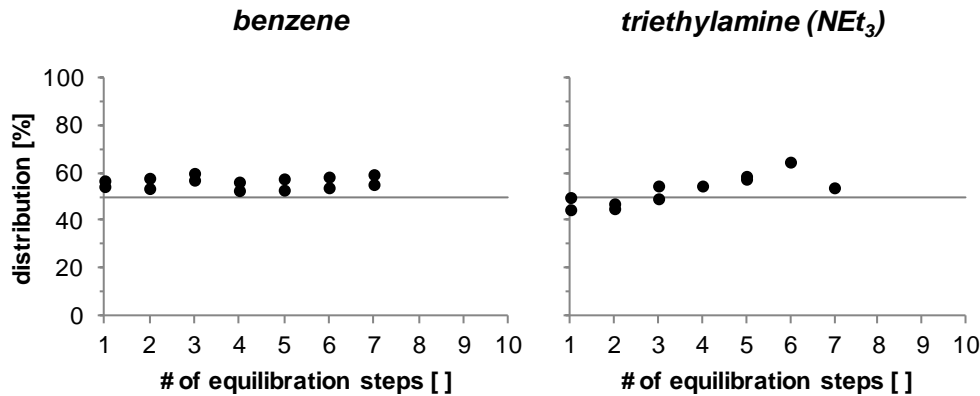

trichloromethane $\left(\mathrm{CHCl}_{3}\right)$

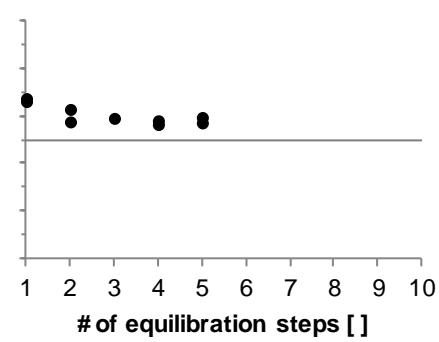

Figure S1. Distribution of benzene, triethylamine and trichloromethane during water-air partitioning. Points that lie above the 50:50 distribution line indicate higher proportion of substance into the gas phase. while points that lie below that line show higher proportion in the liquid phase.
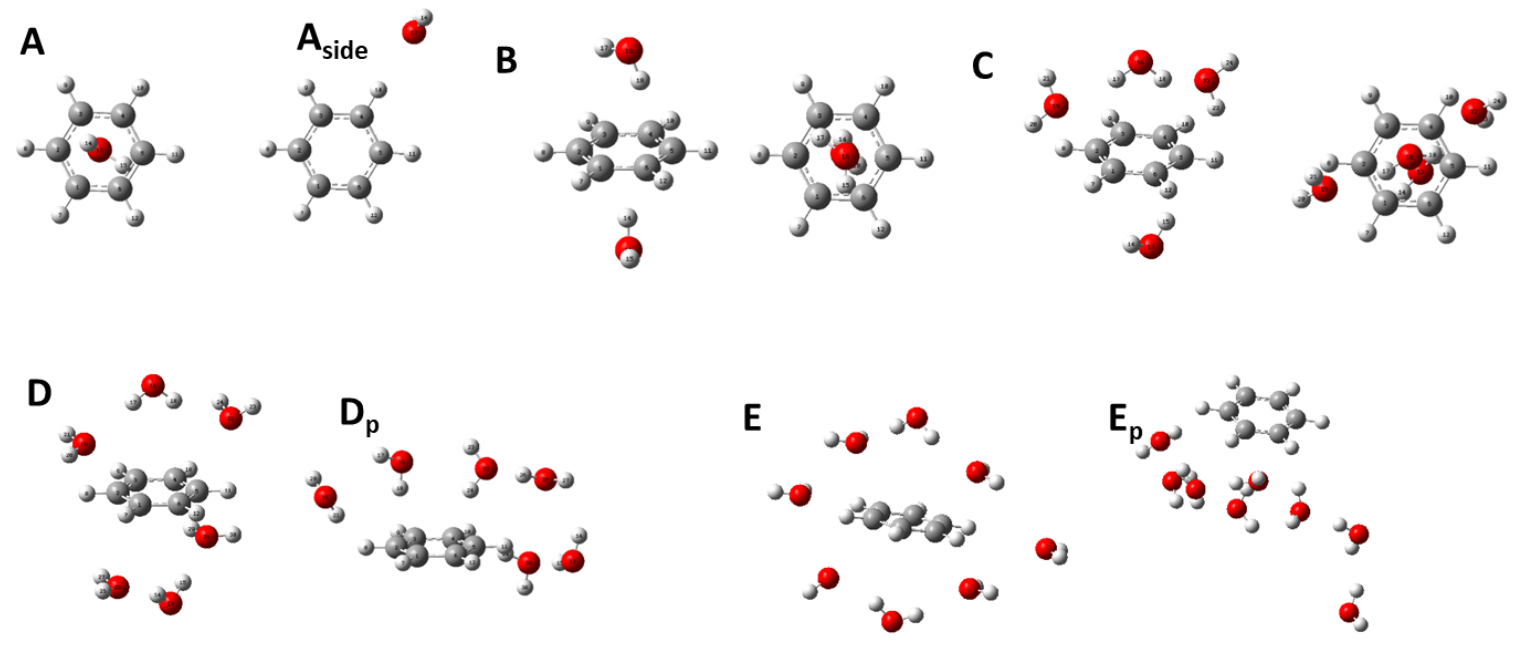

Figure S2. Initial geometries of benzene-water clusters prepared manually by placing water molecules in proximity to the solute. P - denotes structure after initial preoptimization with the PM3 method. Side - denotes structure in which water was in equatorial position. i.e. on a side of the benzene ring. Models A to E consist of 1, 2, 4, 6, and 8 water molecules. respectively. 

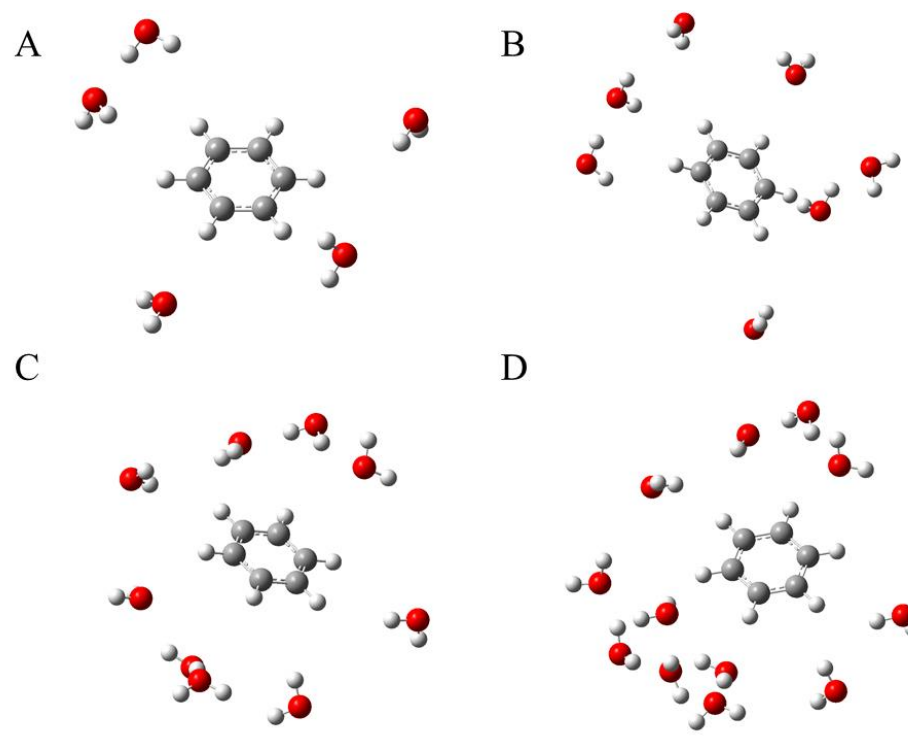

D

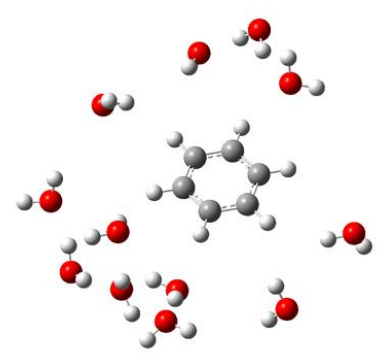

Figure S3. Initial geometries of benzene-water clusters prepared by cutting out solvent molecules from larger water box structure. Models A to D consist of 5, 7, 9, 12 water molecules. respectively.

A

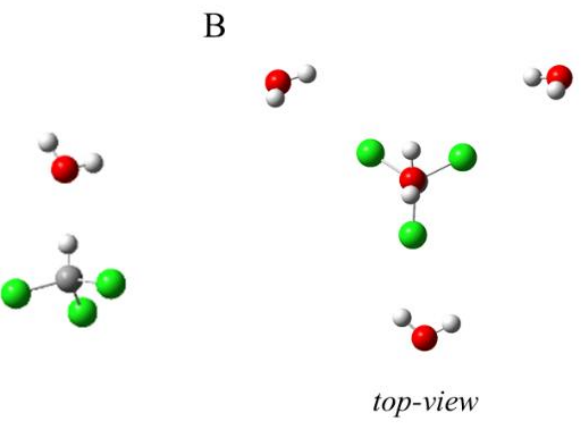

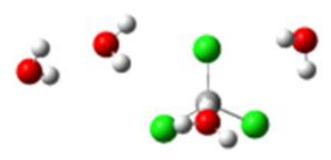

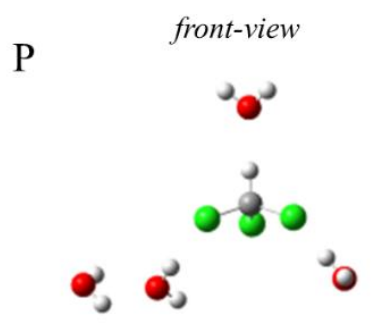

top-view

Figure S4. Initial geometries of trichloromethane-water clusters prepared manually by placing water molecules in proximity to the solute. Models A and B contain 1 and 4 water molecules, respectively. $\mathrm{P}$ - denotes structure after initial preoptimization with the PM3 method. For the latter front and top view is presented in respect to $\mathrm{C}-\mathrm{H}$ trichloromethane bond. 
A

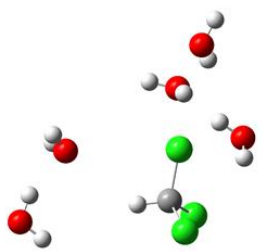

$\mathrm{D}$

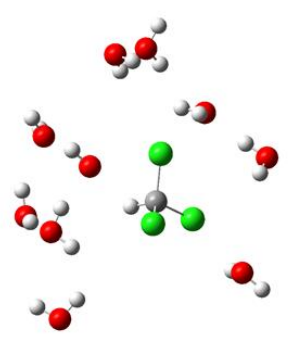

B

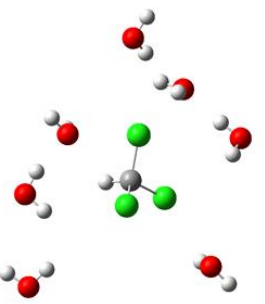

$\mathrm{E}$

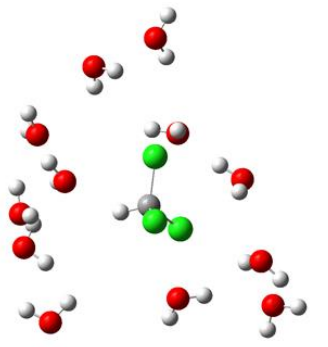

C

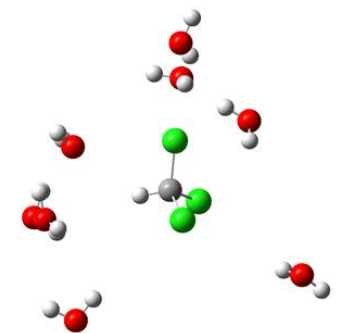

$\mathrm{F}$

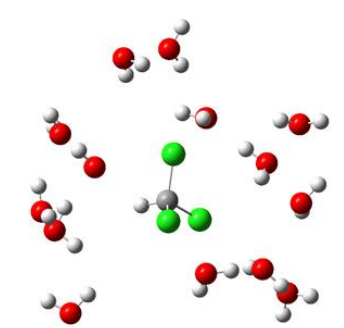

Figure S5. Initial geometries of trichloromethane-water clusters prepared by cutting out solvent molecules larger water box structure. Models A to F contain 5, 7, 8, 1, 12, and 14 water molecules, respectively.

A

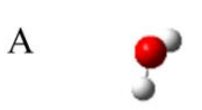

B

B

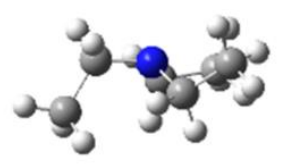

$\mathrm{C}$
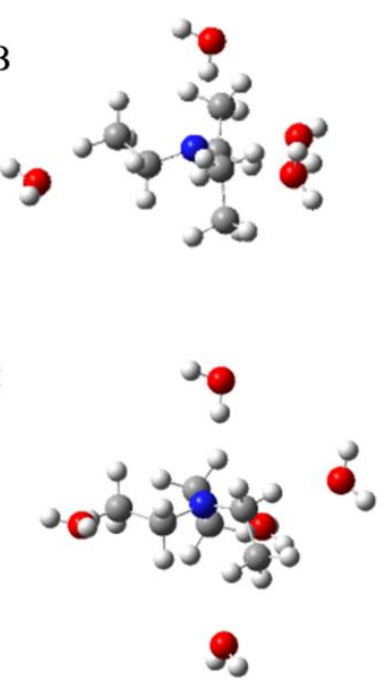

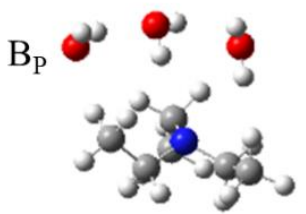

0

$\mathrm{C}_{\mathrm{P}}$
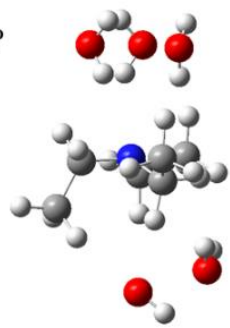

Figure S6. Initial geometries of triethylamine-water clusters prepared manually by placing water molecules in proximity to the solute. Models A. B and C contain 1, 4, and 5 water molecules, respectively. $\mathrm{P}$ - denotes structure after initial preoptimization with the PM3 method. 
A

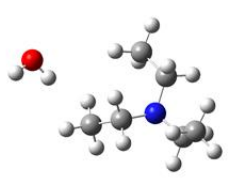

$\mathrm{D}$

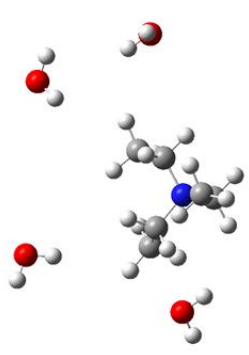

B

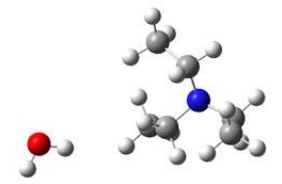

$\mathrm{E}$

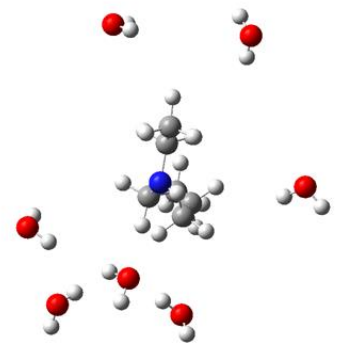

$\mathrm{C}$

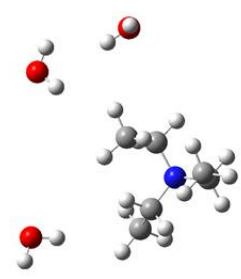

$\mathrm{F}$

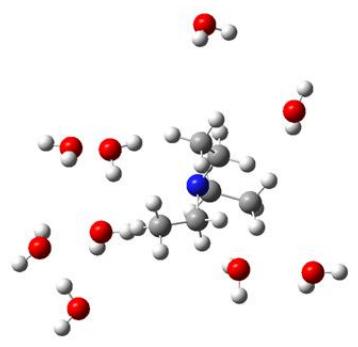

Figure S7. Initial geometries of triethylamine-water clusters prepared by cutting out solvent molecules from larger water box structure. Models A to F contain 1, 2, 3, 4, 7, and 9 water molecules, respectively.

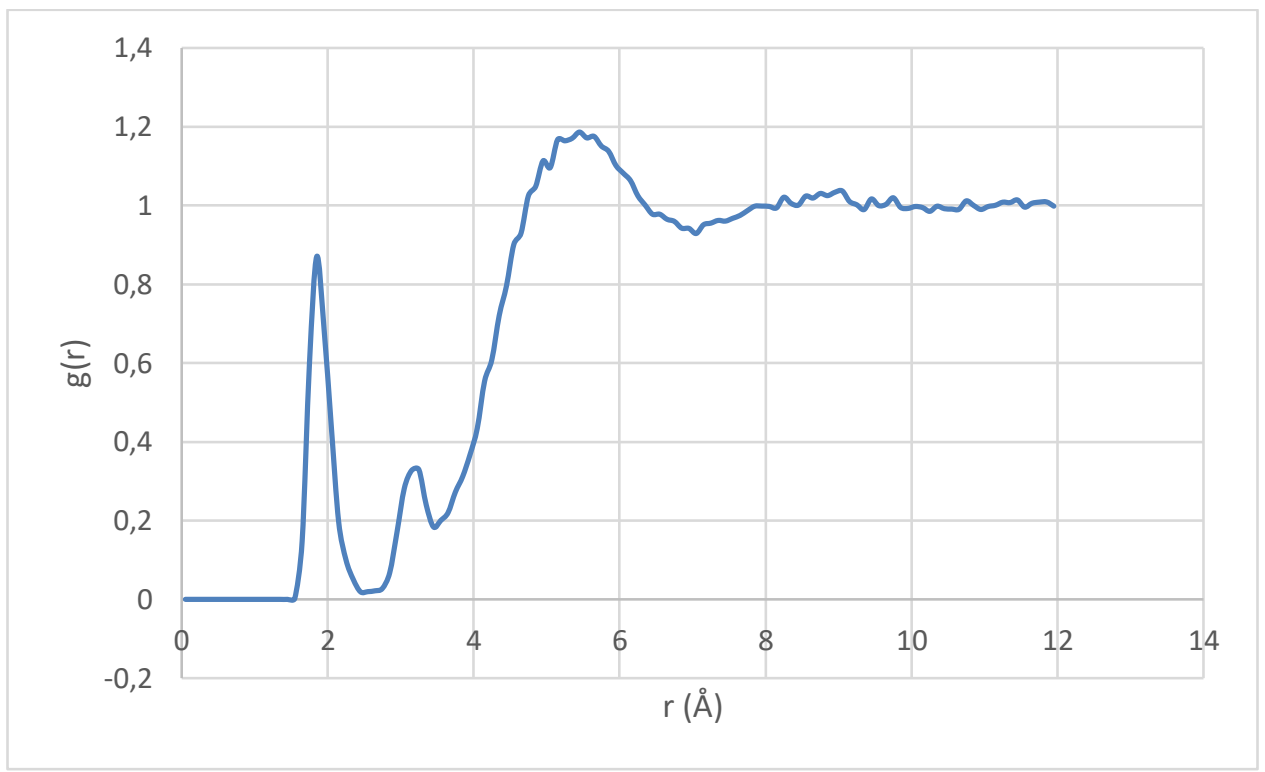

Figure S8. Radial distribution functions between the nitrogen of TEA and the one of the hydrogen atoms of water. 
A

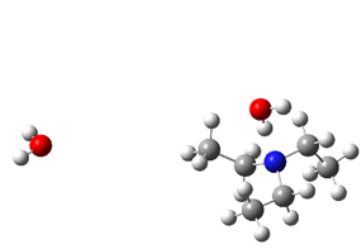

$\mathrm{A}_{\mathrm{p}}$

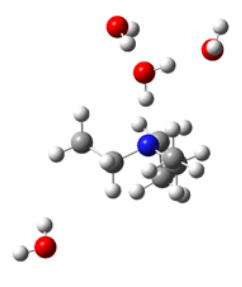

$\mathrm{B}_{\mathrm{P}}$

B

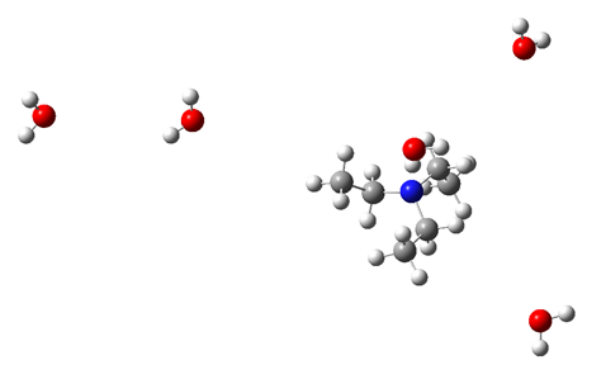

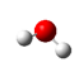
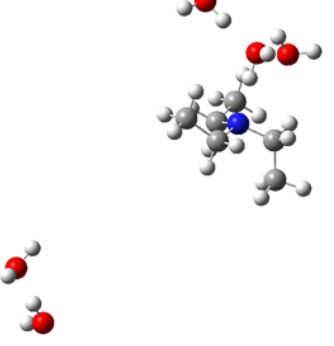

Figure S9. Geometries of triethylamine-water mixed solvent models on structures prepared manually. A and B consisting of 4 and 5 water molecules, respectively optimized in presence of PCM at B3LYP/6$31+\mathrm{G}(\mathrm{d}, \mathrm{p})$ theory level. $\mathrm{P}-$ structures obtained with preoptimization step with the PM3 method. 


\section{References:}

(1) Higuera-Padilla, A. R.; Kock, F. V. C.; Batista, A. A.; Colnago, L. A. A Straightforward Catalytic Approach to Obtain Deuterated Chloroform at Room Temperature. Magn. Reson. Chem. 2020, 58 (10), 917-920. https://doi.org/10.1002/mrc.5066.

(2) Darden, T.; York, D.; Pedersen, L. Particle Mesh Ewald: An N·log(N) Method for Ewald Sums in Large Systems. J. Chem. Phys. 1993. https://doi.org/10.1063/1.464397.

(3) Krzemińska, A.; Paneth, P.; Moliner, V.; Świderek, K. Binding Isotope Effects as a Tool for Distinguishing Hydrophobic and Hydrophilic Binding Sites of HIV-1 RT. J. Phys. Chem. B 2015, 119 (3), 917-927. https://doi.org/10.1021/jp506119h.

(4) Field, M. J.; Albe, M.; Bret, C.; Proust-De Martin, F.; Thomas, A. The Dynamo Library for Molecular Simulations Using Hybrid Quantum Mechanical and Molecular Mechanical Potentials. J. Comput. Chem. 2000, 21 (12), 1088-1100. https://doi.org/10.1002/1096987X(200009)21:12<1088::AID-JCC5>3.0.CO;2-8.

(5) Jeannottat, S.; Hunkeler, D. Chlorine and Carbon Isotopes Fractionation during Volatilization and Diffusive Transport of Trichloroethene in the Unsaturated Zone. Environ. Sci. Technol. 2012, 46 (6), 3169-3176. https://doi.org/10.1021/es203547p.

(6) Nguyen, H. V. L.; Kannengießer, R.; Stahl, W. Microwave Survey of the Conformational Landscape Exhibited by the Propeller Molecule Triethyl Amine. Phys. Chem. Chem. Phys. 2012, 14 (33), 11753-11758. https://doi.org/10.1039/C2CP41385J. 
Geometries of optimized models used in the study

Benzene-Water cluster models prepared manually optimized at B2PLYP-D3/6-311+G(2df,2p) theory level.

Benzene - 1 Water

$\begin{array}{rrrr}\mathrm{C} & -0.60598 & 0.41240 & 1.33746 \\ \mathrm{C} & -0.36420 & -0.93668 & 1.09108 \\ \mathrm{C} & -0.38288 & -1.42031 & -0.21505 \\ \mathrm{C} & -0.64354 & -0.55478 & -1.27417 \\ \mathrm{C} & -0.88673 & 0.79300 & -1.02704 \\ \mathrm{C} & -0.86774 & 1.27656 & 0.27854 \\ \mathrm{H} & -0.58588 & 0.78814 & 2.35004 \\ \mathrm{H} & -0.15930 & -1.60766 & 1.91271 \\ \mathrm{H} & -0.19185 & -2.46627 & -0.40652 \\ \mathrm{H} & -0.65285 & -0.92929 & -2.28738 \\ \mathrm{H} & -1.09016 & 1.46429 & -1.84895 \\ \mathrm{H} & -1.05579 & 2.32321 & 0.46993 \\ \mathrm{O} & 2.73272 & 0.25755 & -0.11471 \\ \mathrm{H} & 1.88712 & -0.16212 & 0.07027 \\ \mathrm{H} & 2.49332 & 1.10827 & -0.48734\end{array}$

Benzene - 2 Water molecules

$\begin{array}{lrrr}\mathrm{C} & 0.13870 & 1.16807 & -0.71393 \\ \mathrm{C} & -0.00932 & -0.05539 & -1.36311 \\ \mathrm{C} & -0.14831 & -1.22502 & -0.61962 \\ \mathrm{C} & -0.14006 & -1.17088 & 0.77278 \\ \mathrm{C} & 0.00898 & 0.05198 & 1.42153 \\ \mathrm{C} & 0.14874 & 1.22130 & 0.67843 \\ \mathrm{H} & 0.24710 & 2.07516 & -1.29046 \\ \mathrm{H} & -0.01638 & -0.09701 & -2.44221 \\ \mathrm{H} & -0.26417 & -2.17382 & -1.12303 \\ \mathrm{H} & -0.25427 & -2.07753 & 1.34892 \\ \mathrm{H} & 0.01656 & 0.09362 & 2.50077 \\ \mathrm{H} & 0.26986 & 2.16965 & 1.18147 \\ \mathrm{O} & -3.39940 & 0.03096 & -0.08529 \\ \mathrm{H} & -2.48698 & -0.26267 & -0.16253 \\ \mathrm{H} & -3.33237 & 0.92955 & 0.24323 \\ \mathrm{O} & 3.40004 & -0.02313 & -0.08699 \\ \mathrm{H} & 2.48650 & 0.26889 & -0.15677 \\ \mathrm{H} & 3.33662 & -0.92876 & 0.22234\end{array}$


Benzene - 4 Water molecules

$\begin{array}{rrrr}\mathrm{C} & -0.94539 & 0.25616 & -1.42592 \\ \mathrm{C} & -0.21673 & -0.63129 & -0.63965 \\ \mathrm{C} & -0.25390 & -0.52343 & 0.74985 \\ \mathrm{C} & -1.02087 & 0.47335 & 1.35032 \\ \mathrm{C} & -1.74819 & 1.36539 & 0.56205 \\ \mathrm{C} & -1.70974 & 1.25639 & -0.82650 \\ \mathrm{H} & -0.92330 & 0.16870 & -2.50261 \\ \mathrm{H} & 0.38794 & -1.40518 & -1.08777 \\ \mathrm{H} & 0.32776 & -1.21323 & 1.34252 \\ \mathrm{H} & -1.05653 & 0.55353 & 2.42723 \\ \mathrm{H} & -2.34616 & 2.13518 & 1.02809 \\ \mathrm{H} & -2.27869 & 1.94235 & -1.43761 \\ \mathrm{O} & -3.77108 & -1.53181 & -0.02669 \\ \mathrm{H} & -3.15942 & -2.25889 & 0.10592 \\ \mathrm{H} & -3.20338 & -0.76004 & -0.10813 \\ \mathrm{O} & 3.64762 & 0.09890 & -0.37155 \\ \mathrm{H} & 3.85817 & 0.20592 & -1.30069 \\ \mathrm{H} & 3.06457 & 0.84469 & -0.14696 \\ \mathrm{O} & 2.35688 & -2.32709 & 0.26385 \\ \mathrm{H} & 3.00797 & -2.95787 & 0.57444 \\ \mathrm{H} & 2.85836 & -1.51792 & 0.06344 \\ \mathrm{O} & 1.80491 & 2.09140 & 0.38548 \\ \mathrm{H} & 0.99518 & 1.59967 & 0.57068 \\ \mathrm{H} & 1.52984 & 2.83250 & -0.15820 \\ & & & \end{array}$

\section{Benzene - 6 Water molecules}

$\begin{array}{lrrr}\mathrm{C} & 1.16263 & -1.11094 & -1.13292 \\ \mathrm{C} & 0.49399 & -1.08903 & 0.08771 \\ \mathrm{C} & 1.19633 & -0.90806 & 1.27521 \\ \mathrm{C} & 2.57885 & -0.74083 & 1.24073 \\ \mathrm{C} & 3.25312 & -0.75651 & 0.02051 \\ \mathrm{C} & 2.54493 & -0.94420 & -1.16563 \\ \mathrm{H} & 0.59640 & -1.24356 & -2.04315 \\ \mathrm{H} & -0.57870 & -1.19603 & 0.11092 \\ \mathrm{H} & 0.65689 & -0.88213 & 2.21064 \\ \mathrm{H} & 3.12989 & -0.59598 & 2.15911 \\ \mathrm{H} & 4.32567 & -0.62556 & -0.00562 \\ \mathrm{H} & 3.06969 & -0.95611 & -2.11033 \\ \mathrm{O} & -0.91025 & 2.28193 & 1.18109 \\ \mathrm{H} & 0.03644 & 2.27783 & 0.92028 \\ \mathrm{H} & -1.03248 & 3.03886 & 1.75772 \\ \mathrm{O} & -2.03654 & -0.70142 & -2.29491 \\ \mathrm{H} & -2.50697 & -1.01469 & -1.51550 \\ \mathrm{H} & -1.72520 & 0.18272 & -2.05958 \\ \mathrm{O} & -3.23803 & -1.81451 & 0.23090 \\ \mathrm{H} & -4.18932 & -1.94415 & 0.26356 \\ \mathrm{H} & -2.85460 & -2.68743 & 0.35335 \\ \mathrm{O} & -1.08959 & 1.98909 & -1.58986 \\ \mathrm{H} & -1.31321 & 2.20018 & -0.66685\end{array}$




$\begin{array}{rrrr}\mathrm{H} & -1.53465 & 2.64361 & -2.13227 \\ \mathrm{O} & 1.44809 & 2.23325 & -0.16476 \\ \mathrm{H} & 0.92383 & 2.07941 & -0.96256 \\ \mathrm{H} & 1.98038 & 1.43659 & -0.05667 \\ \mathrm{O} & -1.96105 & -0.16790 & 2.40311 \\ \mathrm{H} & -1.60851 & 0.63228 & 1.99172 \\ \mathrm{H} & -2.45588 & -0.61197 & 1.70694\end{array}$

\section{Benzene - 6 Water molecules preoptimized with PM3}

$\begin{array}{rrrr}\mathrm{C} & -3.13808 & -0.31642 & 0.62179 \\ \mathrm{C} & -3.08773 & -0.18376 & -0.76436 \\ \mathrm{C} & -1.85715 & -0.17371 & -1.41868 \\ \mathrm{C} & -0.67719 & -0.29094 & -0.68861 \\ \mathrm{C} & -0.72757 & -0.42093 & 0.69836 \\ \mathrm{C} & -1.95716 & -0.43716 & 1.35236 \\ \mathrm{H} & -4.09221 & -0.32688 & 1.12904 \\ \mathrm{H} & -4.00239 & -0.08975 & -1.33174 \\ \mathrm{H} & -1.81833 & -0.06690 & -2.49296 \\ \mathrm{H} & 0.27979 & -0.26606 & -1.18900 \\ \mathrm{H} & 0.19284 & -0.50269 & 1.25585 \\ \mathrm{H} & -1.99502 & -0.53497 & 2.42794 \\ \mathrm{O} & 2.39638 & -1.78640 & 0.32352 \\ \mathrm{H} & 2.51545 & -1.37345 & -0.54046 \\ \mathrm{H} & 1.72342 & -2.47032 & 0.20177 \\ \mathrm{O} & 1.26958 & 2.45099 & 0.15580 \\ \mathrm{H} & 0.31807 & 2.62923 & 0.12312 \\ \mathrm{H} & 1.49587 & 2.04411 & -0.68789 \\ \mathrm{O} & -1.57350 & 2.92834 & 0.08382 \\ \mathrm{H} & -2.01669 & 2.07441 & 0.03648 \\ \mathrm{H} & -1.93124 & 3.35809 & 0.86375 \\ \mathrm{O} & 3.29717 & 0.78759 & 1.17318 \\ \mathrm{H} & 2.52728 & 1.37807 & 1.18191 \\ \mathrm{H} & 2.94705 & -0.10671 & 1.29058 \\ \mathrm{O} & 2.81934 & 0.48278 & -1.48753 \\ \mathrm{H} & 3.21863 & 0.66370 & -0.60791 \\ \mathrm{H} & 3.50461 & 0.65244 & -2.13670 \\ \mathrm{O} & 0.10848 & -3.49657 & -0.09737 \\ \mathrm{H} & -0.57103 & -2.82275 & 0.01065 \\ \mathrm{H} & -0.16638 & -4.23593 & 0.44902 \\ & & & \end{array}$


Trichloromethane-Water cluster models prepared manually optimized at B3LYP/6-31+G(d,p) theory level.

Trichloromethane - 4 Water molecules preoptimized with PM3

$\begin{array}{lrrr}\mathrm{C} & 1.85903 & 0.00995 & 0.05501 \\ \mathrm{H} & 1.18349 & 0.05654 & 0.91039 \\ \mathrm{Cl} & 2.78065 & -1.51733 & 0.14944 \\ \mathrm{Cl} & 0.83085 & 0.03723 & -1.43330 \\ \mathrm{Cl} & 2.93951 & 1.42862 & 0.09530 \\ \mathrm{O} & -0.39653 & 0.09271 & 2.17733 \\ \mathrm{H} & -1.21911 & 0.01629 & 1.65263 \\ \mathrm{H} & -0.55684 & -0.33839 & 3.02426 \\ \mathrm{O} & -4.63587 & 1.42302 & -0.04065 \\ \mathrm{H} & -4.98474 & 2.06712 & -0.66735 \\ \mathrm{H} & -5.08398 & 0.56940 & -0.21064 \\ \mathrm{O} & -4.85952 & -1.30459 & -0.37295 \\ \mathrm{H} & -5.24334 & -2.02142 & 0.14528 \\ \mathrm{H} & -3.90894 & -1.26269 & -0.15260 \\ \mathrm{O} & -2.36178 & -0.07909 & 0.18738 \\ \mathrm{H} & -1.70299 & -0.01191 & -0.51950 \\ \mathrm{H} & -2.97531 & 0.68412 & 0.08401\end{array}$

Trichloromethane-Water cluster models prepared by cutting out solvent molecules from water box optimized at B3LYP/6-31+G(d,p) theory level.

Trichloromethane - 5 Water molecules

$\begin{array}{lrrr}\mathrm{C} & -1.72300 & -0.26990 & -0.06320 \\ \mathrm{H} & -0.75927 & -0.58135 & -0.48192 \\ \mathrm{Cl} & -2.79610 & -1.68232 & 0.07458 \\ \mathrm{Cl} & -1.38054 & 0.42427 & 1.57771 \\ \mathrm{Cl} & -2.42308 & 0.98584 & -1.13067 \\ \mathrm{O} & 2.83027 & 1.45353 & -0.84935 \\ \mathrm{H} & 2.19229 & 2.02975 & -0.36839 \\ \mathrm{H} & 3.18371 & 1.96425 & -1.58784 \\ \mathrm{O} & 0.91535 & 2.78808 & 0.60297 \\ \mathrm{H} & 0.36638 & 3.50238 & 0.25527 \\ \mathrm{H} & 0.29537 & 2.12186 & 0.94348\end{array}$




$\begin{array}{rrrr}\mathrm{O} & 4.53294 & -0.32260 & 0.48825 \\ \mathrm{H} & 3.99182 & 0.38642 & 0.08196 \\ \mathrm{H} & 4.88720 & 0.03013 & 1.31237 \\ \mathrm{O} & 1.03583 & -0.87939 & -1.12758 \\ \mathrm{H} & 1.60799 & -1.59914 & -0.78973 \\ \mathrm{H} & 1.63221 & -0.11891 & -1.22660 \\ \mathrm{O} & 3.01085 & -2.58771 & -0.05758 \\ \mathrm{H} & 2.88974 & -3.20213 & 0.67508 \\ \mathrm{H} & 3.64379 & -1.90158 & 0.24430\end{array}$

Trichloromethane - 7 Water molecules

$\begin{array}{lrrr}\mathrm{C} & -2.49961 & -0.16832 & 0.27401 \\ \mathrm{H} & -1.62719 & 0.48777 & 0.29526 \\ \mathrm{Cl} & -3.04756 & -0.45959 & 1.94423 \\ \mathrm{Cl} & -3.76746 & 0.61927 & -0.70737 \\ \mathrm{Cl} & -1.97029 & -1.71862 & -0.49731 \\ \mathrm{O} & 4.32730 & -2.30621 & -0.12329 \\ \mathrm{H} & 4.26758 & -1.88061 & 0.76507 \\ \mathrm{H} & 5.24469 & -2.58138 & -0.23601 \\ \mathrm{O} & 0.45682 & -0.22929 & -2.53062 \\ \mathrm{H} & -0.19881 & -0.88267 & -2.23998 \\ \mathrm{H} & 0.29115 & -0.07230 & -3.46950 \\ \mathrm{O} & 2.72711 & 1.10509 & 0.69514 \\ \mathrm{H} & 3.01078 & 0.73918 & -0.18627 \\ \mathrm{H} & 2.82156 & 2.07525 & 0.65883 \\ \mathrm{O} & 3.26643 & -0.22075 & -1.57591 \\ \mathrm{H} & 3.65577 & -1.05127 & -1.21819 \\ \mathrm{H} & 2.41492 & -0.44362 & -1.98498 \\ \mathrm{O} & 0.10928 & 1.49511 & -0.19929 \\ \mathrm{H} & 0.91870 & 1.18675 & 0.26757 \\ \mathrm{H} & 0.22156 & 1.16170 & -1.10737 \\ \mathrm{O} & 4.01107 & -0.82005 & 2.16180 \\ \mathrm{H} & 3.47704 & -1.12508 & 2.90451 \\ \mathrm{H} & 3.55683 & -0.02722 & 1.79100 \\ \mathrm{O} & 1.81746 & 3.73951 & 0.18545 \\ \mathrm{H} & 1.58312 & 4.55569 & 0.64168 \\ \mathrm{H} & 0.98655 & 3.26239 & -0.00417 \\ & & & \end{array}$

Trichloromethane - 8 Water molecules

$\begin{array}{llrr}\mathrm{C} & 1.75740 & -0.01067 & -0.00777 \\ \mathrm{H} & 0.73374 & -0.39407 & 0.01681 \\ \mathrm{Cl} & 1.76511 & 1.54456 & -0.89159 \\ \mathrm{Cl} & 2.29460 & 0.22294 & 1.69245 \\ \mathrm{Cl} & 2.78086 & -1.22740 & -0.83554\end{array}$




$\begin{array}{rrrr}\mathrm{O} & -1.06592 & -1.22352 & 0.40457 \\ \mathrm{H} & -1.33393 & -1.98305 & -0.16809 \\ \mathrm{H} & -0.94324 & -1.57015 & 1.31006 \\ \mathrm{O} & -2.10674 & -3.13101 & -1.24666 \\ \mathrm{H} & -1.67117 & -3.40703 & -2.06159 \\ \mathrm{H} & -2.94218 & -2.67334 & -1.51101 \\ \mathrm{O} & 5.66668 & 1.16336 & -0.97236 \\ \mathrm{H} & 5.15617 & 0.34367 & -1.00624 \\ \mathrm{H} & 5.00404 & 1.86424 & -0.92689 \\ \mathrm{O} & -3.21049 & 0.40231 & -0.16062 \\ \mathrm{H} & -2.42690 & -0.10031 & 0.16886 \\ \mathrm{H} & -3.66103 & 0.80504 & 0.61037 \\ \mathrm{O} & -0.29474 & -1.90696 & 3.02806 \\ \mathrm{H} & -0.22057 & -2.78181 & 3.42897 \\ \mathrm{H} & 0.57229 & -1.48700 & 3.11642 \\ \mathrm{O} & -3.13661 & 3.11435 & -0.85023 \\ \mathrm{H} & -2.95340 & 2.15238 & -0.88961 \\ \mathrm{H} & -2.30686 & 3.56855 & -1.03702 \\ \mathrm{O} & -4.25401 & -1.55153 & -1.76728 \\ \mathrm{H} & -5.16686 & -1.78954 & -1.56856 \\ \mathrm{H} & -4.02496 & -0.77618 & -1.19965 \\ \mathrm{O} & -4.28398 & 2.33258 & 1.53760 \\ \mathrm{H} & -5.18812 & 2.59114 & 1.74963 \\ \mathrm{H} & -3.97452 & 2.92314 & 0.81919\end{array}$

Trichloromethane-Water cluster models prepared by cutting out solvent molecules from water box optimized at B3LYP/6-311+G(2df,2p) theory level.

Trichloromethane - 5 Water molecules

$\begin{array}{lrrr}\mathrm{C} & -1.67118 & -0.27703 & -0.06337 \\ \mathrm{H} & -0.73450 & -0.55019 & -0.54733 \\ \mathrm{Cl} & -2.73833 & -1.68760 & 0.00089 \\ \mathrm{Cl} & -1.26251 & 0.27491 & 1.60369 \\ \mathrm{Cl} & -2.41353 & 1.05591 & -0.98675 \\ \mathrm{O} & 2.80420 & 1.53306 & -0.80070 \\ \mathrm{H} & 2.11997 & 2.07016 & -0.35016 \\ \mathrm{H} & 3.21823 & 2.09660 & -1.45935 \\ \mathrm{O} & 0.74523 & 2.81513 & 0.54366 \\ \mathrm{H} & 0.12610 & 3.34719 & 0.03472 \\ \mathrm{H} & 0.20556 & 2.11883 & 0.94408 \\ \mathrm{O} & 4.43539 & -0.31065 & 0.58308 \\ \mathrm{H} & 3.91308 & 0.40447 & 0.17597\end{array}$




$\begin{array}{rrrr}\mathrm{H} & 4.70843 & 0.00866 & 1.44599 \\ \mathrm{O} & 1.06002 & -0.83153 & -1.32027 \\ \mathrm{H} & 1.61630 & -1.54454 & -0.95748 \\ \mathrm{H} & 1.64120 & -0.05942 & -1.34160 \\ \mathrm{O} & 2.95374 & -2.59428 & -0.12093 \\ \mathrm{H} & 2.72515 & -3.15707 & 0.62231 \\ \mathrm{H} & 3.54321 & -1.90115 & 0.23122\end{array}$

Trichloromethane - 7 Water molecules

$\begin{array}{lrrr}\mathrm{C} & -1.83277 & -0.08257 & -0.08128 \\ \mathrm{H} & -0.96974 & -0.26143 & -0.72463 \\ \mathrm{Cl} & -3.06793 & -1.30964 & -0.39835 \\ \mathrm{Cl} & -1.27807 & -0.15387 & 1.62018 \\ \mathrm{Cl} & -2.46583 & 1.55745 & -0.45370 \\ \mathrm{O} & 1.26182 & -3.31409 & -1.34080 \\ \mathrm{H} & 1.33583 & -3.27501 & -0.36386 \\ \mathrm{H} & 0.77152 & -4.11215 & -1.54907 \\ \mathrm{O} & 0.07926 & 3.56168 & 0.60430 \\ \mathrm{H} & -0.60753 & 2.97708 & 0.25583 \\ \mathrm{H} & -0.01174 & 3.52442 & 1.56071 \\ \mathrm{O} & 3.14242 & 0.65257 & -0.97906 \\ \mathrm{H} & 3.00894 & 1.61544 & -0.82796 \\ \mathrm{H} & 3.80367 & 0.56689 & -1.67104 \\ \mathrm{O} & 2.64964 & 3.27199 & -0.39635 \\ \mathrm{H} & 2.80100 & 3.99873 & -1.00538 \\ \mathrm{H} & 1.73967 & 3.38695 & -0.05599 \\ \mathrm{O} & 3.56546 & -0.99889 & 1.24997 \\ \mathrm{H} & 3.46124 & -0.37533 & 0.50608 \\ \mathrm{H} & 3.99637 & -0.51680 & 1.95875 \\ \mathrm{O} & 0.60076 & -0.61952 & -1.81119 \\ \mathrm{H} & 0.79151 & -1.57542 & -1.77094 \\ \mathrm{H} & 1.43467 & -0.18459 & -1.58071 \\ \mathrm{O} & 1.48725 & -2.84408 & 1.37877 \\ \mathrm{H} & 0.71470 & -2.33382 & 1.63916 \\ \mathrm{H} & 2.23455 & -2.21404 & 1.41326\end{array}$

Trichloromethane - 10 Water molecules

$\begin{array}{llll}\mathrm{C} & -3.12664 & 0.24138 & 0.09637 \\ \mathrm{H} & -2.14026 & 0.68952 & 0.06139\end{array}$




$\begin{array}{lrrr}\mathrm{Cl} & -4.34067 & 1.53535 & -0.00519 \\ \mathrm{Cl} & -3.26688 & -0.87005 & -1.29922 \\ \mathrm{Cl} & -3.26856 & -0.65545 & 1.62926 \\ \mathrm{O} & -0.06372 & 1.10954 & -0.00749 \\ \mathrm{H} & 0.34934 & 1.99531 & -0.07828 \\ \mathrm{H} & 0.32534 & 0.69131 & 0.78691 \\ \mathrm{O} & 0.72028 & -0.33228 & -2.14565 \\ \mathrm{H} & 0.40371 & 0.23223 & -1.39339 \\ \mathrm{H} & 0.29140 & -0.00040 & -2.93925 \\ \mathrm{O} & 0.07628 & -2.88525 & -1.11756 \\ \mathrm{H} & -0.87298 & -2.93301 & -0.97393 \\ \mathrm{H} & 0.23154 & -2.04107 & -1.58105 \\ \mathrm{O} & 3.90022 & 0.59481 & 1.77327 \\ \mathrm{H} & 2.98549 & 0.41900 & 2.05891 \\ \mathrm{H} & 4.19473 & -0.24208 & 1.37329 \\ \mathrm{O} & 1.17571 & -0.19811 & 2.14652 \\ \mathrm{H} & 0.72651 & -0.28220 & 2.99176 \\ \mathrm{H} & 1.32606 & -1.12754 & 1.80604 \\ \mathrm{O} & 3.87303 & 2.29580 & -0.27586 \\ \mathrm{H} & 3.91918 & 1.71895 & 0.53568 \\ \mathrm{H} & 4.68964 & 2.80103 & -0.30675 \\ \mathrm{O} & 1.71792 & -2.56798 & 1.12629 \\ \mathrm{H} & 1.13634 & -2.77807 & 0.36853 \\ \mathrm{H} & 2.61402 & -2.46866 & 0.76136 \\ \mathrm{O} & 3.56311 & -0.10427 & -1.89799 \\ \mathrm{H} & 3.68352 & 0.78541 & -1.52701 \\ \mathrm{H} & 2.61079 & -0.18535 & -2.08589 \\ \mathrm{O} & 1.34683 & 3.45730 & -0.36596 \\ \mathrm{H} & 1.27295 & 4.22904 & 0.20022 \\ \mathrm{H} & 2.28032 & 3.16690 & -0.32500 \\ \mathrm{O} & 4.26144 & -1.72050 & 0.13087 \\ \mathrm{H} & 5.01254 & -2.28007 & -0.08128 \\ \mathrm{H} & 4.04473 & -1.19846 & -0.68448 \\ & & & \end{array}$

Trichloromethane - 12 Water molecules

$\begin{array}{lrrr}\mathrm{C} & 2.64758 & -1.65879 & 0.20522 \\ \mathrm{H} & 1.59990 & -1.57907 & -0.06633 \\ \mathrm{Cl} & 3.62162 & -0.92434 & -1.10156 \\ \mathrm{Cl} & 2.89182 & -0.78206 & 1.74147 \\ \mathrm{Cl} & 3.04719 & -3.38081 & 0.39132 \\ \mathrm{O} & -2.80470 & -2.75008 & -1.29193 \\ \mathrm{H} & -2.66927 & -3.06396 & -2.19082 \\ \mathrm{H} & -3.45711 & -2.02880 & -1.34856 \\ \mathrm{O} & -0.40808 & -1.24551 & -0.46478 \\ \mathrm{H} & -1.10737 & -1.87826 & -0.68638 \\ \mathrm{H} & -0.47635 & -0.53584 & -1.15226 \\ \mathrm{O} & 1.80902 & 2.07057 & -2.39178 \\ \mathrm{H} & 2.49413 & 1.52669 & -1.99261 \\ \mathrm{H} & 1.79268 & 2.90660 & -1.88593 \\ \mathrm{O} & -4.17100 & 1.17820 & 0.99177\end{array}$




$\begin{array}{rrrr}\mathrm{H} & -4.53439 & 0.45217 & 0.46091 \\ \mathrm{H} & -3.41168 & 0.79093 & 1.45205 \\ \mathrm{O} & -5.04896 & -1.11568 & -0.61707 \\ \mathrm{H} & -5.84950 & -0.97114 & -1.12917 \\ \mathrm{H} & -5.20482 & -1.91162 & -0.06840 \\ \mathrm{O} & -2.17946 & 2.54954 & -0.43498 \\ \mathrm{H} & -3.04777 & 2.34273 & -0.04120 \\ \mathrm{H} & -1.82127 & 3.34402 & -0.00830 \\ \mathrm{O} & -4.80137 & -3.58208 & 0.57193 \\ \mathrm{H} & -4.58981 & -3.77872 & 1.48822 \\ \mathrm{H} & -3.97256 & -3.65552 & 0.07164 \\ \mathrm{O} & -1.28626 & 0.60677 & 1.42049 \\ \mathrm{H} & -1.47769 & 1.25170 & 0.71184 \\ \mathrm{H} & -0.94367 & -0.17011 & 0.93171 \\ \mathrm{O} & -0.43253 & 4.37806 & 0.94685 \\ \mathrm{H} & -0.65249 & 5.15259 & 1.47142 \\ \mathrm{H} & -0.08510 & 3.70135 & 1.58300 \\ \mathrm{O} & -0.68067 & 0.81082 & -2.20609 \\ \mathrm{H} & 0.18008 & 1.25271 & -2.35254 \\ \mathrm{H} & -1.23711 & 1.47065 & -1.76289 \\ \mathrm{O} & 1.63267 & 4.48249 & -0.98151 \\ \mathrm{H} & 1.54076 & 5.24778 & -1.55421 \\ \mathrm{H} & 0.90029 & 4.53023 & -0.33800 \\ \mathrm{O} & 0.42057 & 2.40148 & 2.56788 \\ \mathrm{H} & 1.33867 & 2.12877 & 2.48861 \\ \mathrm{H} & -0.11845 & 1.63282 & 2.26994 \\ & & & \end{array}$

Trichloromethane - 14 Water molecules

$\begin{array}{lrrr}\mathrm{C} & 3.76093 & -0.13182 & 0.05776 \\ \mathrm{H} & 3.16667 & -0.05665 & 0.96419 \\ \mathrm{Cl} & 4.24328 & -1.82763 & -0.16390 \\ \mathrm{Cl} & 2.71150 & 0.39927 & -1.31446 \\ \mathrm{Cl} & 5.16645 & 0.93383 & 0.19706 \\ \mathrm{O} & 2.11118 & -0.11398 & 2.68886 \\ \mathrm{H} & 2.61469 & -0.01579 & 3.50142 \\ \mathrm{H} & 1.24767 & 0.33430 & 2.85741 \\ \mathrm{O} & -0.10376 & -2.14107 & 2.80190 \\ \mathrm{H} & -0.32552 & -2.57844 & 1.96563 \\ \mathrm{H} & 0.80568 & -1.82662 & 2.70691 \\ \mathrm{O} & 0.80747 & 3.66469 & -1.32981 \\ \mathrm{H} & 1.55589 & 3.09159 & -1.14409 \\ \mathrm{H} & 0.21205 & 3.15707 & -1.90914 \\ \mathrm{O} & -4.92282 & 1.42059 & 1.74889 \\ \mathrm{H} & -4.02231 & 1.74660 & 1.95459 \\ \mathrm{H} & -5.37882 & 2.16124 & 1.34128 \\ \mathrm{O} & -0.41185 & 0.61487 & 3.27772 \\ \mathrm{H} & -0.68883 & -0.30792 & 3.14444 \\ \mathrm{H} & -1.08125 & 1.18851 & 2.86923 \\ \mathrm{O} & -5.28997 & -0.93787 & 0.37990 \\ \mathrm{H} & -5.12552 & -0.11814 & 0.89429\end{array}$




$\begin{array}{rrrr}\mathrm{H} & -6.17046 & -1.23181 & 0.62773 \\ \mathrm{O} & -2.37877 & 2.44181 & 2.26807 \\ \mathrm{H} & -2.41761 & 3.11206 & 2.95654 \\ \mathrm{H} & -2.08695 & 2.91020 & 1.43995 \\ \mathrm{O} & -1.68003 & 3.69372 & 0.02923 \\ \mathrm{H} & -1.92455 & 3.24343 & -0.79681 \\ \mathrm{H} & -0.73301 & 3.86718 & -0.10779 \\ \mathrm{O} & -4.17022 & -1.35743 & -2.30986 \\ \mathrm{H} & -3.91823 & -2.17955 & -1.84800 \\ \mathrm{H} & -4.69045 & -0.90644 & -1.62909 \\ \mathrm{O} & -0.76316 & -3.43068 & 0.27397 \\ \mathrm{H} & -0.49212 & -4.35300 & 0.26024 \\ \mathrm{H} & -0.34122 & -3.00486 & -0.49373 \\ \mathrm{O} & -3.62983 & -3.24301 & -0.28996 \\ \mathrm{H} & -2.70979 & -3.28773 & 0.00928 \\ \mathrm{H} & -4.05759 & -2.54657 & 0.23113 \\ \mathrm{O} & 0.37295 & -1.98807 & -1.91059 \\ \mathrm{H} & 0.64313 & -2.43737 & -2.71796 \\ \mathrm{H} & 1.11410 & -1.41546 & -1.66617 \\ \mathrm{O} & -1.79889 & -0.14946 & -2.91186 \\ \mathrm{H} & -1.14138 & -0.73880 & -2.52125 \\ \mathrm{H} & -2.67815 & -0.54932 & -2.71031 \\ \mathrm{O} & -1.46838 & 2.47094 & -2.55509 \\ \mathrm{H} & -1.79096 & 2.88557 & -3.35946 \\ \mathrm{H} & -1.60302 & 1.49418 & -2.67591 \\ & & & \end{array}$

Triethylamine-Water cluster models prepared manually optimized with PCM calculations at the B3LYP/6-31+G(d,p) theory level.

\section{Triethylamine - 1 Water}

$\begin{array}{rrrr}\mathrm{N} & -0.20436 & -0.04349 & 0.03451 \\ \mathrm{C} & -1.46203 & -0.76359 & 0.34668 \\ \mathrm{H} & -1.40081 & -1.74165 & -0.14369 \\ \mathrm{H} & -1.46685 & -0.95427 & 1.42672 \\ \mathrm{C} & -0.11707 & 1.24129 & 0.76045 \\ \mathrm{H} & -0.40240 & 1.04013 & 1.79958 \\ \mathrm{H} & -0.84561 & 1.96759 & 0.36326 \\ \mathrm{C} & -2.77791 & -0.07560 & -0.04830 \\ \mathrm{H} & -3.61867 & -0.73470 & 0.19414 \\ \mathrm{H} & -2.92777 & 0.86430 & 0.49166 \\ \mathrm{H} & -2.82162 & 0.13671 & -1.12177 \\ \mathrm{C} & 1.27339 & 1.87728 & 0.74035 \\ \mathrm{H} & 1.26873 & 2.77668 & 1.36526 \\ \mathrm{H} & 2.03107 & 1.19131 & 1.13213 \\ \mathrm{H} & 1.57112 & 2.18115 & -0.26778 \\ \mathrm{C} & 0.02964 & 0.08887 & -1.42730 \\ \mathrm{H} & -0.71065 & -0.52199 & -1.95391\end{array}$




$\begin{array}{rrrr}\mathrm{H} & -0.14866 & 1.12628 & -1.74618 \\ \mathrm{C} & 1.42569 & -0.36091 & -1.86970 \\ \mathrm{H} & 1.53052 & -0.23611 & -2.95365 \\ \mathrm{H} & 2.21559 & 0.21860 & -1.38430 \\ \mathrm{H} & 1.58669 & -1.41747 & -1.63248 \\ \mathrm{O} & 1.62879 & -1.81897 & 1.27231 \\ \mathrm{H} & 1.29473 & -1.91085 & 2.17392 \\ \mathrm{H} & 1.01449 & -1.17355 & 0.82395\end{array}$

Triethylamine - 4 Water molecules

$\begin{array}{rrrr}\mathrm{N} & 0.23495 & -0.08630 & 0.15559 \\ \mathrm{C} & 1.10026 & 1.07354 & -0.16618 \\ \mathrm{H} & 0.55428 & 1.97392 & 0.13723 \\ \mathrm{H} & 1.98298 & 1.00913 & 0.48150 \\ \mathrm{C} & 0.92837 & -1.36913 & -0.08679 \\ \mathrm{H} & 1.92818 & -1.27939 & 0.35354 \\ \mathrm{H} & 1.06848 & -1.54284 & -1.16672 \\ \mathrm{C} & 1.55718 & 1.22068 & -1.62576 \\ \mathrm{H} & 2.14546 & 2.13875 & -1.73091 \\ \mathrm{H} & 2.18777 & 0.38509 & -1.94445 \\ \mathrm{H} & 0.70845 & 1.28979 & -2.31438 \\ \mathrm{C} & 0.22380 & -2.58296 & 0.52032 \\ \mathrm{H} & 0.84009 & -3.47472 & 0.36436 \\ \mathrm{H} & 0.06910 & -2.45646 & 1.59651 \\ \mathrm{H} & -0.74720 & -2.77026 & 0.05186 \\ \mathrm{C} & -1.09663 & -0.00434 & -0.49971 \\ \mathrm{H} & -1.19588 & 0.98222 & -0.96382 \\ \mathrm{H} & -1.15927 & -0.73445 & -1.31977 \\ \mathrm{C} & -2.26939 & -0.20212 & 0.46580 \\ \mathrm{H} & -3.21717 & -0.13437 & -0.08069 \\ \mathrm{H} & -2.23211 & -1.17680 & 0.95986 \\ \mathrm{H} & -2.26871 & 0.57072 & 1.24121 \\ \mathrm{O} & 0.09216 & 0.24051 & 2.97008 \\ \mathrm{H} & 1.01530 & 0.32092 & 3.24266 \\ \mathrm{H} & 0.11617 & 0.09858 & 1.98305 \\ \mathrm{O} & 3.11281 & 6.24734 & -0.17442 \\ \mathrm{H} & 3.41104 & 6.92529 & 0.44685 \\ \mathrm{H} & 2.93509 & 6.72396 & -0.99653 \\ \mathrm{O} & -9.10978 & -0.32230 & -0.77084 \\ \mathrm{H} & -9.79578 & 0.31224 & -1.01856 \\ \mathrm{H} & -9.58782 & -1.13398 & -0.55350 \\ \mathrm{O} & 5.34954 & -4.46010 & -0.76334 \\ \mathrm{H} & 5.06381 & -5.38362 & -0.77318 \\ \mathrm{H} & 6.31365 & -4.49729 & -0.82304\end{array}$

Triethylamine - 4 Water molecules preoptimized with PM3 


\begin{tabular}{|c|c|c|c|}
\hline $\mathrm{N}$ & -0.20319 & 0.13579 & 0.58529 \\
\hline $\mathrm{C}$ & 0.21124 & -0.72473 & 1.71973 \\
\hline $\mathrm{H}$ & 0.46130 & -1.71033 & 1.31585 \\
\hline $\mathrm{H}$ & 1.14741 & -0.30409 & 2.10395 \\
\hline C & -0.34498 & 1.54974 & 1.00169 \\
\hline $\mathrm{H}$ & 0.50098 & 1.77378 & 1.66057 \\
\hline $\mathrm{H}$ & -1.26063 & 1.69345 & 1.59922 \\
\hline $\mathrm{C}$ & -0.78443 & -0.88788 & 2.87839 \\
\hline $\mathrm{H}$ & -0.34030 & -1.53182 & 3.64509 \\
\hline $\mathrm{H}$ & -1.02789 & 0.06931 & 3.34999 \\
\hline $\mathrm{H}$ & -1.71969 & -1.35658 & 2.55594 \\
\hline C & -0.34076 & 2.54819 & -0.15890 \\
\hline $\mathrm{H}$ & -0.34363 & 3.56691 & 0.24306 \\
\hline $\mathrm{H}$ & 0.55279 & 2.43075 & -0.78004 \\
\hline $\mathrm{H}$ & -1.22048 & 2.45017 & -0.80154 \\
\hline $\mathrm{C}$ & -1.41371 & -0.34731 & -0.11545 \\
\hline $\mathrm{H}$ & -2.29728 & -0.28534 & 0.54251 \\
\hline $\mathrm{H}$ & -1.59833 & 0.33844 & -0.94634 \\
\hline $\mathrm{C}$ & -1.28781 & -1.76388 & -0.67825 \\
\hline $\mathrm{H}$ & -2.16421 & -1.98460 & -1.29627 \\
\hline H & -0.39700 & -1.86221 & -1.30726 \\
\hline $\mathrm{H}$ & -1.24047 & -2.52562 & 0.10565 \\
\hline 0 & 2.01018 & -0.13193 & -1.13064 \\
\hline $\mathrm{H}$ & 1.22069 & -0.00313 & -0.53032 \\
\hline $\mathrm{H}$ & 2.64667 & 0.57956 & -0.92207 \\
\hline 0 & 3.14263 & -2.62114 & -1.20956 \\
\hline H & 3.38851 & -2.86861 & -0.30927 \\
\hline H & 2.73325 & -1.72410 & -1.13690 \\
\hline 0 & -5.52866 & 0.35618 & -1.57831 \\
\hline $\mathrm{H}$ & -4.79508 & -0.27358 & -1.58563 \\
\hline H & -6.19689 & -0.03224 & -2.15894 \\
\hline 0 & 3.92028 & 1.91135 & -0.54200 \\
\hline $\mathrm{H}$ & 3.94623 & 2.64915 & -1.16712 \\
\hline $\mathrm{H}$ & 4.83369 & 1.59976 & -0.4763 \\
\hline
\end{tabular}

\section{Triethylamine - 5 Water molecules}

$\begin{array}{rrrr}\mathrm{N} & 1.46942 & -0.33866 & 0.37728 \\ \mathrm{C} & 1.98411 & 0.93806 & -0.17277 \\ \mathrm{H} & 1.32631 & 1.73337 & 0.19554 \\ \mathrm{H} & 2.96868 & 1.11168 & 0.27802 \\ \mathrm{C} & 2.36385 & -1.47129 & 0.05724 \\ \mathrm{H} & 3.38635 & -1.14148 & 0.27472 \\ \mathrm{H} & 2.32655 & -1.71047 & -1.01858 \\ \mathrm{C} & 2.10527 & 1.04233 & -1.70093 \\ \mathrm{H} & 2.45056 & 2.04662 & -1.96956 \\ \mathrm{H} & 2.82615 & 0.32520 & -2.10523 \\ \mathrm{H} & 1.14400 & 0.87779 & -2.19915 \\ \mathrm{C} & 2.07033 & -2.74236 & 0.85513 \\ \mathrm{H} & 2.82386 & -3.50078 & 0.61770 \\ \mathrm{H} & 2.10223 & -2.55218 & 1.93260 \\ \mathrm{H} & 1.09166 & -3.16496 & 0.60812 \\ & & & \end{array}$




$\begin{array}{rrrr}\mathrm{C} & 0.05060 & -0.58725 & 0.01095 \\ \mathrm{H} & -0.34848 & 0.31400 & -0.46547 \\ \mathrm{H} & -0.01023 & -1.38384 & -0.74487 \\ \mathrm{C} & -0.84130 & -0.93351 & 1.20738 \\ \mathrm{H} & -1.86883 & -1.10759 & 0.86763 \\ \mathrm{H} & -0.49906 & -1.83323 & 1.72593 \\ \mathrm{H} & -0.85759 & -0.11159 & 1.93040 \\ \mathrm{O} & 1.80906 & 0.20686 & 3.14016 \\ \mathrm{H} & 2.72854 & 0.49423 & 3.21028 \\ \mathrm{H} & 1.67125 & -0.01646 & 2.17778 \\ \mathrm{O} & 3.66555 & 5.90940 & 0.36863 \\ \mathrm{H} & 3.49705 & 6.37315 & 1.19998 \\ \mathrm{H} & 4.04005 & 6.57974 & -0.21870 \\ \mathrm{O} & -12.06710 & -1.03703 & 0.79888 \\ \mathrm{H} & -12.57306 & -1.74405 & 0.37611 \\ \mathrm{H} & -12.52124 & -0.87205 & 1.63621 \\ \mathrm{O} & 6.80983 & -3.00980 & -2.06274 \\ \mathrm{H} & 6.99636 & -3.82987 & -2.53945 \\ \mathrm{H} & 7.60515 & -2.47150 & -2.17336 \\ \mathrm{O} & -7.80616 & 1.41659 & -2.66796 \\ \mathrm{H} & -8.33025 & 0.62042 & -2.50670 \\ \mathrm{H} & -7.94836 & 1.97045 & -1.88849 \\ & & & \end{array}$

Triethylamine - 5 Water molecules preoptimized with PM3

$\begin{array}{lrrr}\text { N } & -1.41718 & -0.67369 & -0.00427 \\ \mathrm{C} & -1.76623 & -2.10055 & 0.19835 \\ \mathrm{H} & -2.19370 & -2.19938 & 1.20066 \\ \mathrm{H} & -2.57636 & -2.32602 & -0.50436 \\ \mathrm{C} & -1.04181 & -0.40125 & -1.41047 \\ \mathrm{H} & -1.73898 & -0.96346 & -2.04113 \\ \mathrm{H} & -0.03231 & -0.78743 & -1.62962 \\ \mathrm{C} & -0.64052 & -3.13098 & 0.02134 \\ \mathrm{H} & -1.04535 & -4.13595 & 0.18224 \\ \mathrm{H} & -0.21201 & -3.10354 & -0.98544 \\ \mathrm{H} & 0.17091 & -2.98363 & 0.74125 \\ \mathrm{C} & -1.11155 & 1.07742 & -1.80091 \\ \mathrm{H} & -0.91400 & 1.17654 & -2.87358 \\ \mathrm{H} & -2.10338 & 1.49304 & -1.59712 \\ \mathrm{H} & -0.36952 & 1.68647 & -1.27629 \\ \mathrm{C} & -0.39062 & -0.17393 & 0.93720 \\ \mathrm{H} & 0.57932 & -0.66767 & 0.75588 \\ \mathrm{H} & -0.24309 & 0.88669 & 0.71755 \\ \mathrm{C} & -0.77244 & -0.31333 & 2.41175 \\ \mathrm{H} & -0.02889 & 0.20451 & 3.02624 \\ \mathrm{H} & -1.75024 & 0.13699 & 2.61171 \\ \mathrm{H} & -0.80062 & -1.35605 & 2.74188 \\ \mathrm{O} & -3.86099 & 0.58576 & 0.58800 \\ \mathrm{H} & -2.98821 & 0.15816 & 0.35171 \\ \mathrm{H} & -4.48897 & 0.36418 & -0.12711 \\ \mathrm{O} & -3.76854 & 3.24340 & 1.22071\end{array}$




$\begin{array}{rrrr}\mathrm{H} & -3.78193 & 2.29112 & 0.95436 \\ \mathrm{H} & -3.35419 & 3.71295 & 0.48582 \\ \mathrm{O} & 8.88982 & 1.60969 & -0.63488 \\ \mathrm{H} & 7.96991 & 1.65008 & -0.33846 \\ \mathrm{H} & 8.85698 & 1.73493 & -1.59351 \\ \mathrm{O} & -5.73360 & -0.02933 & -1.48143 \\ \mathrm{H} & -6.41615 & -0.67238 & -1.24398 \\ \mathrm{H} & -6.21276 & 0.73365 & -1.83366 \\ \mathrm{O} & 10.34914 & -0.64662 & 0.23758 \\ \mathrm{H} & 11.07442 & -0.30881 & 0.77851 \\ \mathrm{H} & 9.85169 & 0.14338 & -0.05705\end{array}$

Triethylamine-Water cluster models prepared by cutting out solvent molecules from water box optimized with PCM calculations at the B3LYP/6-31+G(d,p) theory level.

Triethylamine - 1 Water

$\begin{array}{rrrr}\mathrm{N} & 0.89661 & 0.42171 & -0.33287 \\ \mathrm{C} & 2.01488 & 0.11690 & 0.57765 \\ \mathrm{H} & 2.72124 & -0.52689 & 0.04326 \\ \mathrm{H} & 2.54197 & 1.06105 & 0.75965 \\ \mathrm{C} & -0.00048 & 1.45816 & 0.20168 \\ \mathrm{H} & 0.61332 & 2.15718 & 0.78068 \\ \mathrm{H} & -0.73872 & 1.03349 & 0.90775 \\ \mathrm{C} & 1.66689 & -0.53124 & 1.93036 \\ \mathrm{H} & 2.58362 & -0.67532 & 2.51317 \\ \mathrm{H} & 0.99235 & 0.09748 & 2.52131 \\ \mathrm{H} & 1.19612 & -1.51232 & 1.80853 \\ \mathrm{C} & -0.73643 & 2.24692 & -0.88561 \\ \mathrm{H} & -1.35132 & 3.02945 & -0.42687 \\ \mathrm{H} & -0.02280 & 2.72422 & -1.56557 \\ \mathrm{H} & -1.40277 & 1.61466 & -1.48086 \\ \mathrm{C} & 0.15675 & -0.76153 & -0.79560 \\ \mathrm{H} & -0.31723 & -1.29933 & 0.04788 \\ \mathrm{H} & -0.66278 & -0.40554 & -1.42669 \\ \mathrm{C} & 0.99935 & -1.74238 & -1.61361 \\ \mathrm{H} & 0.35458 & -2.52607 & -2.02558 \\ \mathrm{H} & 1.49169 & -1.23054 & -2.44785 \\ \mathrm{H} & 1.76977 & -2.23550 & -1.01280 \\ \mathrm{O} & -4.13248 & -0.97988 & 0.63330 \\ \mathrm{H} & -3.18753 & -0.82546 & 0.49832 \\ \mathrm{H} & -4.40367 & -0.31449 & 1.28017\end{array}$


Triethylamine - 3 Water molecules

$\begin{array}{rrrr}\mathrm{N} & -1.31414 & -0.48272 & -0.77135 \\ \mathrm{C} & -1.77113 & -1.70369 & -1.45881 \\ \mathrm{H} & -2.77036 & -1.51121 & -1.86322 \\ \mathrm{H} & -1.11445 & -1.84428 & -2.32561 \\ \mathrm{C} & 0.10392 & -0.54959 & -0.38387 \\ \mathrm{H} & 0.62620 & -1.13750 & -1.14685 \\ \mathrm{H} & 0.23953 & -1.08887 & 0.57247 \\ \mathrm{C} & -1.80245 & -3.00601 & -0.63790 \\ \mathrm{H} & -2.13169 & -3.83311 & -1.27691 \\ \mathrm{H} & -0.81310 & -3.26286 & -0.24458 \\ \mathrm{H} & -2.49727 & -2.94468 & 0.20619 \\ \mathrm{C} & 0.77464 & 0.82412 & -0.28838 \\ \mathrm{H} & 1.83361 & 0.70486 & -0.03295 \\ \mathrm{H} & 0.70920 & 1.35345 & -1.24496 \\ \mathrm{H} & 0.32176 & 1.45622 & 0.48198 \\ \mathrm{C} & -2.17097 & -0.06591 & 0.34894 \\ \mathrm{H} & -2.19807 & -0.83242 & 1.14646 \\ \mathrm{H} & -1.71099 & 0.81974 & 0.79714 \\ \mathrm{C} & -3.60103 & 0.29221 & -0.06064 \\ \mathrm{H} & -4.13630 & 0.70790 & 0.79988 \\ \mathrm{H} & -3.60181 & 1.04250 & -0.85912 \\ \mathrm{H} & -4.16799 & -0.57679 & -0.40872 \\ \mathrm{O} & 8.05125 & 1.99887 & -1.10221 \\ \mathrm{H} & 7.25466 & 2.42009 & -0.75202 \\ \mathrm{H} & 7.88868 & 1.04959 & -1.01820 \\ \mathrm{O} & 3.40729 & -2.54510 & 2.56755 \\ \mathrm{H} & 3.21021 & -3.23102 & 3.21967 \\ \mathrm{H} & 2.57183 & -2.39276 & 2.10540 \\ \mathrm{O} & -3.02757 & 4.67211 & 1.08365 \\ \mathrm{H} & -3.03182 & 3.74365 & 0.81415 \\ \mathrm{H} & -3.92860 & 4.98262 & 0.92135 \\ & & & \end{array}$

Triethylamine - 4 Water molecules

$\begin{array}{rrrr}\mathrm{N} & 0.28472 & -1.11192 & -0.06779 \\ \mathrm{C} & 1.00254 & -2.39045 & -0.21694 \\ \mathrm{H} & 2.01046 & -2.26301 & 0.19160 \\ \mathrm{H} & 0.49969 & -3.11454 & 0.43515 \\ \mathrm{C} & -1.14901 & -1.22922 & -0.37819 \\ \mathrm{H} & -1.47064 & -2.23173 & -0.07476 \\ \mathrm{H} & -1.33799 & -1.15691 & -1.46547 \\ \mathrm{C} & 1.10488 & -2.98068 & -1.63548 \\ \mathrm{H} & 1.63332 & -3.93984 & -1.59532 \\ \mathrm{H} & 0.11722 & -3.16284 & -2.07234\end{array}$




$\begin{array}{rrrr}\text { H } & 1.66051 & -2.32621 & -2.31517 \\ \mathrm{C} & -2.01562 & -0.20083 & 0.35488 \\ \mathrm{H} & -3.07206 & -0.36308 & 0.11278 \\ \mathrm{H} & -1.89215 & -0.29491 & 1.43915 \\ \mathrm{H} & -1.76927 & 0.82788 & 0.07300 \\ \mathrm{C} & 0.91010 & 0.01572 & -0.77489 \\ \mathrm{H} & 0.94170 & -0.15703 & -1.86727 \\ \mathrm{H} & 0.26779 & 0.88868 & -0.62579 \\ \mathrm{C} & 2.31354 & 0.36264 & -0.27386 \\ \mathrm{H} & 2.66155 & 1.27992 & -0.76100 \\ \mathrm{H} & 2.31023 & 0.52988 & 0.80893 \\ \mathrm{H} & 3.04394 & -0.42156 & -0.49596 \\ \mathrm{O} & -5.49568 & -1.50679 & 2.60334 \\ \mathrm{H} & -4.64917 & -1.06568 & 2.75701 \\ \mathrm{H} & -5.60524 & -1.50449 & 1.64273 \\ \mathrm{O} & -5.08221 & 2.49904 & -1.55635 \\ \mathrm{H} & -5.16650 & 3.46182 & -1.53385 \\ \mathrm{H} & -4.27378 & 2.30763 & -1.06185 \\ \mathrm{O} & 7.40364 & 0.55272 & 1.14543 \\ \mathrm{H} & 6.74589 & -0.09853 & 1.42467 \\ \mathrm{H} & 8.24337 & 0.22438 & 1.49410 \\ \mathrm{O} & 0.83946 & 4.69380 & 0.39894 \\ \mathrm{H} & 1.13515 & 3.77416 & 0.35964 \\ \mathrm{H} & 1.65270 & 5.21610 & 0.42052 \\ & & & \end{array}$

\section{Triethylamine - 7 Water molecules}

$\begin{array}{rrrr}\text { N } & 0.70918 & 0.30480 & 1.14380 \\ \mathrm{C} & -0.01543 & 0.24478 & 2.43448 \\ \mathrm{H} & -0.99663 & 0.70714 & 2.28864 \\ \mathrm{H} & -0.20318 & -0.81564 & 2.63820 \\ \mathrm{C} & 1.95457 & -0.49304 & 1.18010 \\ \mathrm{H} & 1.73079 & -1.41093 & 1.73443 \\ \mathrm{H} & 2.74462 & 0.03322 & 1.74235 \\ \mathrm{C} & 0.66867 & 0.88089 & 3.65479 \\ \mathrm{H} & 0.03203 & 0.74567 & 4.53586 \\ \mathrm{H} & 1.63661 & 0.41763 & 3.87070 \\ \mathrm{H} & 0.82737 & 1.95623 & 3.52466 \\ \mathrm{C} & 2.48888 & -0.87556 & -0.20281 \\ \mathrm{H} & 3.35849 & -1.53115 & -0.08574 \\ \mathrm{H} & 1.73139 & -1.41277 & -0.78220 \\ \mathrm{H} & 2.81283 & -0.00634 & -0.78307 \\ \mathrm{C} & 0.96208 & 1.68134 & 0.66723 \\ \mathrm{H} & 1.66160 & 2.20740 & 1.33986 \\ \mathrm{H} & 1.46703 & 1.59857 & -0.29892 \\ \mathrm{C} & -0.30188 & 2.52196 & 0.48042 \\ \mathrm{H} & -0.03796 & 3.46755 & -0.00446 \\ \mathrm{H} & -1.02817 & 2.00544 & -0.15570 \\ \mathrm{H} & -0.78872 & 2.76638 & 1.42934 \\ \mathrm{O} & 2.95232 & -5.15404 & -0.51148 \\ \mathrm{H} & 2.34746 & -4.47696 & -0.84383\end{array}$




$\begin{array}{lrrr}\mathrm{H} & 3.76939 & -4.67846 & -0.30915 \\ \mathrm{O} & 6.50481 & -0.97613 & -2.16706 \\ \mathrm{H} & 6.94078 & -0.45738 & -2.85653 \\ \mathrm{H} & 5.65517 & -0.53740 & -2.02425 \\ \mathrm{O} & -7.88796 & -0.59719 & -1.78829 \\ \mathrm{H} & -7.00272 & -0.22421 & -1.58394 \\ \mathrm{H} & -8.52377 & 0.06833 & -1.49615 \\ \mathrm{O} & -5.35873 & 0.42980 & -1.21084 \\ \mathrm{H} & -4.80439 & -0.12907 & -0.62612 \\ \mathrm{H} & -4.81358 & 0.63191 & -1.98274 \\ \mathrm{O} & 3.28721 & 4.53760 & -2.19092 \\ \mathrm{H} & 2.55110 & 4.21694 & -1.65236 \\ \mathrm{H} & 2.92302 & 5.27696 & -2.69624 \\ \mathrm{O} & -3.80866 & -1.16706 & 0.51347 \\ \mathrm{H} & -4.10106 & -2.09022 & 0.52632 \\ \mathrm{H} & -3.85942 & -0.86784 & 1.43316 \\ \mathrm{O} & -1.07868 & -1.04227 & -0.63770 \\ \mathrm{H} & -1.95042 & -1.04701 & -0.20711 \\ \mathrm{H} & -0.46783 & -0.55539 & -0.02423\end{array}$

